June, 1995

HUTP-95/A021

\title{
A LATTICE CONSTRUCTION OF CHIRAL GAUGE THEORIES
}

\author{
Pilar Hernández \\ and \\ Raman Sundrumi \\ Lyman Laboratory of Physics \\ Harvard University \\ Cambridge, MA 02138, USA
}

\begin{abstract}
We formulate chiral gauge theories non-perturbatively, using two different cuttoffs for the fermions and gauge bosons. We use a lattice with spacing $b$ to regulate the gauge fields in standard fashion, while computing the chiral fermion determinant on a finer lattice with spacing $f \ll b$. This determinant is computed in the background of $f$-lattice gauge fields, obtained by gauge-covariantly interpolating $b$ lattice gauge fields. The notorious doublers that plague lattice theories containing fermions are decoupled by the addition of a Wilson term. In chiral theories such a term breaks gauge invariance explicitly. However, the advantage of the two-cutoff regulator is that gauge invariance can be restored to $O\left(f^{2} / b^{2}\right)$ by a one-loop subtraction of calculable local gauge field counterterms. We show that the only obstruction to this procedure is the presence of an uncancelled gauge anomaly among the fermion representations. We conclude that for practical purposes, it suffices to choose $f / b \sim b / L$, where $L^{4}$ is the physical volume of the system. In our construction it is simple to prove the Adler-Bardeen theorem for anomalies in global currents to all orders. The related subject of fermion number violation is also studied. Finally, we discuss the prospects for improving the efficiency of our algorithm.
\end{abstract}

*hernandez@huhepl.harvard.edu. Junior Fellow, Harvard Society of Fellows. Supported by the Milton Fund of Harvard University and by the National Science Foundation under grant NSF-PHYS-92-18167

${ }^{\dagger}$ sundrum@huhepl.harvard.edu. Supported by NSF under grant NSF-PHYS-92-18167 


\section{Introduction}

Particle theories which go beyond the Standard Model are likely to depend on the non-perturbative dynamics of chiral gauge theories. It is well appreciated that an important step for theoretical and computer investigations of these theories is their formulation on the lattice. The major obstacle to accomplishing this goal is the seeming difficulty of avoiding 'fermion doubling', while at the same time maintaining chiral gauge invariance [1]. In this paper we describe a non-perturbative lattice construction, which we show leads in the continuum limit to Green functions satisfying the Ward identities of a given chiral gauge theory, precisely when gauge anomalies cancel among the fermion representations.

Several of the tools we employ exist in some form in the earlier literature on the subject. The most important of these is the idea of coupling continuum fermions to interpolated lattice gauge fields as a step towards maintaining chiral symmetry. Reference [2] contains early work on this topic. More recently it has been used in the context of maintaining ungauged chiral symmetry by 't Hooft [3]. Also, the authors of [4] and very recently [5] and [6] have used a gauge field interpolation to the continuum in defining a fermion determinant for chiral gauge theories.

Our chiral gauge theory construction makes use of two lattices: the gauge fields live on the links of a regular lattice with spacing $b$, while the fermions live on a much finer lattice subdividing the $b$-lattice, with spacing $f \ll b$. The gauge field action is the standard one. For the fermion-gauge boson interaction we gauge-covariantly interpolate the $b$-lattice gauge fields to $f$ lattice gauge fields and couple these to the fermions. If the interpolation is 'smooth' enough this will result in a regulated theory in which the gauge boson momenta are cutoff above $1 / b$, while the fermion momenta are at most of order $1 / f$. The utility of having these two cutoffs is easy to understand on very general grounds and we outline these next.

The Nielsen-Ninomiya theorem [7] strongly suggests that in order to remove unwanted doublers from the spectrum of light fermion modes on the lattice, the chiral gauge symmetry must be broken explicitly. Resigning ourselves to this conclusion, there is a simple lattice fermion regulator which eliminates the doubler-modes with a Wilson term and results in a gauge non-invariant fermion determinant. However, this breakage of gauge symmetry can be cured by renormalization. The approach of restoring chiral symmetries through the addition of counterterms is well-known to work in lattice QCD [8]. The case of chiral gauge theories has been considered by 
the Rome group [9] [10], and also by the authors of [11]. They propose to recover a gauge invariant theory by a non-perturbative tuning of counterterms. The major advantage of our approach is that the use of two cutoffs allows us to recover a symmetric continuum limit after an analytic one-loop subtraction.

Due to the Wilson term, the fermionic one-loop effective action is not gauge invariant. Under a gauge transformation $\delta_{x}^{a}$ on the lattice, it transforms as,

$$
\delta_{x}^{a} \Gamma_{0}=L^{a}(x)+O\left(f^{2}\right),
$$

where $L^{a}(x)$ is a sum of local, dimension $\leq 4$ operators made from the gauge fields, while the $O\left(f^{2}\right)$ terms may be non-local in the gauge fields. This form is not surprising. Any sensible regulator with short-distance cutoff $f$ should give gauge invariant results in the $f \rightarrow 0$ limit, except in the presence of ultraviolet divergences, which are sensitive to the details of the cutoff procedure. The local nature of these ultraviolet divergences underlies the locality of $L^{a}$. Any other violation of gauge symmetry must vanish as $f \rightarrow 0$.

The fact that the large violations of gauge invariance are local suggests that they can be subtracted by defining a 'renormalized' fermion effective action,

$$
\begin{aligned}
& \Gamma_{r}=\Gamma_{0}-S_{c . t .}, \\
& \delta_{x}^{a} S_{c . t .}=L^{a}(x),
\end{aligned}
$$

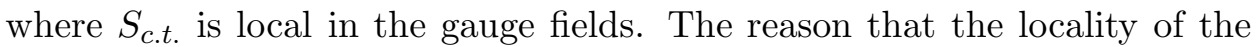
subtractions is important is that the non-local 'finite' parts represent the consequences of one-loop unitarity and must be left intact. Clearly $\Gamma_{r}$ is now gauge invariant up to order $f^{2}$. This procedure provides a nice understanding of chiral gauge anomalies. The (consistent) anomaly [12] is the only term in $L^{a}$ which is not the gauge variation of a local functional. Therefore it cannot be subtracted. Gauge invariance up to $O\left(f^{2}\right)$ can only be restored in theories for which the gauge anomaly cancels among the chiral fermion representations. Obviously, we will assume this to be the case.

If we had exactly cancelled all gauge non-invariance we could proceed to integrate over gauge fields without further anxiety, since the gauge fields are gauge-invariantly regulated on the lattice. But this is not the case, since there remains an $O\left(f^{2}\right)$ non-local violation of gauge invariance. When the fermion determinant is inserted in gauge boson loops, new divergences threaten to overcome the $O\left(f^{2}\right)$ suppression. This is where having two cutoffs makes a crucial difference. The point is that the new gauge boson 
ultraviolet divergences are at most given by powers of $1 / b \ll 1 / f$. Therefore the violations of gauge invariance are suppressed by the small parameter $f^{2} / b^{2}$ to all orders! This is the central mechanism in our construction for reproducing a gauge invariant theory in the continuum limit.

A regulator with two cutoffs, $f \ll b$, may seem strange at first, but it is simple to understand if we imagine integrating down to a theory with a single cutoff, $b$. One could in principle integrate out fermion loop momenta between the scales $1 / f$ and $1 / b$, to yield a theory cut off at $b$, but which contains a series of higher dimensional local interactions resulting from this integration. The new theory is equivalent to the two-cutoff theory for amplitudes with external momenta below $1 / b$. In particular, though gauge invariance is not manifest, the higher-dimensional interactions will compensate the gauge non-invariance of the cutoff procedure to yield amplitudes related by the gauge Ward identities to order $f^{2} / b^{2}$.

The remainder of this paper is organized as follows. In section 2 , we begin with the formal path integral for a chiral gauge theory and explain how the various parts are to be formulated on two lattices, focussing mostly on the fermions. In particular we explain how the loss of gauge invariance in the regulated fermion determinant can be compensated by subtraction of local counterterms. The obstruction provided by gauge anomalies to doing this is emphasized. We also propose a hybrid of this fermion regulator and the standard Wilson regulator for vector-like theories. It separately regulates the real and imaginary parts of the fermion effective action, and enjoys improved gauge invariance properties over the original regulator, which will be important in section 4. Section 3 describes our adaptation of 't Hooft's gauge field interpolation scheme, stressing the important properties it satisfies. In section 4 , we study the scaling of the effective action with $f$, before integration over gauge fields, taking into account the $f$ dependence introduced by the interpolation. We show that the effective action in the hybrid scheme is gauge invariant to $O\left(f^{2}\right)$ and finite in the limit $f \rightarrow 0$. In section 5 , using the results of section 4 , it is shown that the gauge Ward identities (or BRST identities if gauge-fixing is employed) hold to $O\left(f^{2} / b^{2}\right)$ after functional integration over gauge fields. The choice of how small to take $f / b$ in practice is then made. Section 6 deals with anomalies in global currents which occur in chiral theories. The Adler-Bardeen theorem is demonstrated to hold at one loop and then proven to hold to all orders. The second step is made particularly simple by the use of the two-cutoff regulator. Section 7 outlines how the anomalies in global currents translate into the phenomenon of fermion number violation in our construction. Section 8 discusses some 
new ideas for improving computational efficiency. Section 9 provides our conclusions.

\section{Two-Cutoff Lattice Chiral Gauge Theory}

Our target theory is one describing a left-handed fermion with a non-abelian gauge charge. The continuum version which we want to regulate with the lattice is formally given by the generating functional

$$
\begin{aligned}
e^{W\left[J_{\mu}, \bar{\eta}, \eta\right]} & =\int \mathcal{D} A_{\mu} \mathcal{D} \bar{\psi} \mathcal{D} \psi \exp ^{-\int \frac{1}{4} \operatorname{tr} F_{\mu \nu}^{2}+\bar{\psi} i \hat{D} \psi+J_{\mu} A_{\mu}+\bar{\eta} \psi+\bar{\psi} \eta} \\
& =\int \mathcal{D} A_{\mu} e^{-\int \frac{1}{4} \operatorname{tr} F_{\mu \nu}^{2}+J_{\mu} A_{\mu}} e^{\Gamma[A]} e^{-\iint \bar{\eta} G[A] \eta},
\end{aligned}
$$

where

$$
\begin{aligned}
e^{\Gamma[A]} & =\operatorname{det} i \hat{D}, \\
G[A] & =(i \hat{D})^{-1}, \\
\hat{D} & =(\not \partial+i \not A) L+\not \partial R,
\end{aligned}
$$

and $R, L=\frac{1}{2}\left(1 \pm \gamma_{5}\right)$. We see that $\psi_{L}$ transforms (in general reducibly) under the gauge group, while $\psi_{R}$ is taken to be sterile.

The above manipulations are valid assuming that $i \hat{D}$ has no zero-modes, which would leave $G[A]$ ill-defined. However, the occurrence of zero modes in topologically non-trivial gauge field backgrounds is physically important, for example in fermion-number violating processes. For most of this paper we will restrict our attention to fermion number conserving processes. In sections 6 and 7 we show that fermion number violation occurs in our construction and we describe a procedure for defining general fermion number violating amplitudes.

Our lattice construction of the target theory (3) will take the form

$$
e^{W\left[J_{\mu}, \bar{\eta}, \eta\right]}=\int \prod_{s} d U_{\mu}(s) e^{-S_{g}[U]+\sum_{s} J_{\mu} A_{\mu}} e^{\Gamma[U]} e^{-\sum \bar{\eta} G[U] \eta},
$$

where $U_{\mu}(s)=e^{i b A_{\mu}(s)}$ is the gauge field variable on the link $(s, s+\hat{\mu})$ of a regular lattice with spacing $b$. The gauge boson kinetic terms are defined in the usual way on the $b$-lattice,

$$
\begin{gathered}
S_{g}[U]=\frac{2}{g_{0}^{2}} \sum_{s} \sum_{\mu<\nu}\left[I-\frac{1}{2}\left(U_{\mu \nu}+U_{\mu \nu}^{\dagger}\right)\right], \\
U_{\mu \nu} \equiv U_{\mu}(s) U_{\nu}(s+\hat{\mu}) U_{\mu}^{-1}(s+\hat{\nu}) U_{\nu}^{-1}(s) .
\end{gathered}
$$


To define the fermion determinant however, we employ a much finer lattice which subdivides the $b$-lattice, with spacing $f \ll b$, where $b / f$ is an integer. The fermion determinant is then given by the $f$-lattice path integral with action

$$
\begin{aligned}
S_{f}^{\chi w}[\Psi]= & \frac{f^{3}}{2} \sum_{n, \mu}\left(\bar{\Psi}(n) \gamma_{\mu}\left[R+u_{\mu} L\right] \Psi(n+\hat{\mu})-\bar{\Psi}(n+\hat{\mu}) \gamma_{\mu}\left[R+u_{\mu}^{-1} L\right] \Psi(n)\right. \\
& \left.+4 r f^{3} \sum_{n} \bar{\Psi}(n) \Psi(n)-\frac{r f^{3}}{2} \sum_{n, \mu}[\bar{\Psi}(n) \Psi(n+\hat{\mu})+\bar{\Psi}(n+\hat{\mu}) \Psi(n)]\right\},
\end{aligned}
$$

where $n$ denotes the points of the $f$-lattice (as opposed to $s$ for the points of the $b$-lattice). We refer to this as the chiral Wilson $(\chi w)$ lattice regulator to distinguish it from the standard Wilson $(s w)$ regulator for vector-like gauge theories [13]. It is also the regulator used by the Rome group [9] for $f=b$. The action describes a left-handed fermion coupled to a gauge field $u_{\mu}$, and a sterile right-handed fermion. The two chiralities are coupled together by a Wilson term proportional to $r$, which gives the left- and right-handed doubler modes a Dirac mass of order $r / f$ but clearly breaks chiral gauge symmetry explicitly. We take $r=O(1)$. The explicit breaking of gauge invariance is a nuisance but not fatal. As we will show, it can be made small to all orders by the addition of gauge field counterterms determined at one (fermion) loop. Note that we have not added a mass for the fermion. It is not required because the $\chi w$ action possesses a 'shift' symmetry, $\psi_{R} \rightarrow \psi_{R}+\xi$, where $\xi$ is a constant spinor [14. On the other hand, this is not a symmetry of the standard Wilson term of vector-like theories and, as is well-known, mass counterterms are required in that context用.

The $f$-lattice link variables, $u_{\mu}(n)$, are interpolations of the $b$-lattice gauge fields, $U_{\nu}(s)$, which are the real dynamical variables. The one-loop fermion effective action (the logarithm of the fermion determinant) will therefore be a functional of the $b$-lattice gauge fields,

$$
\Gamma[U]=\Gamma[u[U]]
$$

The interpolation $u[U]$ must be carefully chosen so that a (nearly) gauge invariant, lattice rotation and translation invariant, determinant functional of $u_{\mu}$ induces a (nearly) gauge invariant, lattice rotation and translation invariant functional of $U_{\mu}$. Furthermore, the non-locality of $\Gamma[u]$ correctly

\footnotetext{
${ }^{\ddagger}$ In any case, in the two-cutoff theory only a mass of $O\left(f / b^{2}\right)$ would be generated.
} 
describes the effects of virtual massless fermions dictated by one-loop unitarity, and it is essential that this same non-locality be found in the $\Gamma[U]$ induced by the interpolation. In section 3 , we will describe such an interpolation and its properties in detail. For the remainder of this section we will assume that we have such an interpolation procedure and concentrate on compensating the breakage of gauge invariance (in terms of $u_{\mu}$ ) inherent to the $\chi w$ regulator.

We will denote the unsubtracted (gauge non-invariant) $\chi w$ determinant by $\exp \Gamma_{0}[u]$. Consider the effect of an infinitesimal gauge transformation,

$$
u_{\mu}(n) \rightarrow \omega(n) u_{\mu}(n) \omega^{-1}(n+\hat{\mu}),
$$

with $\omega(n) \equiv e^{i \theta^{a}(n) T^{a}}$. We have then,

$$
\begin{aligned}
\delta_{\omega} \Gamma_{0}[u] & =<\delta_{\omega} S_{f}^{\chi w}>_{u}, \\
\delta_{\omega} S_{f}^{\chi w} & =-i 4 f^{3} \sum_{n} \theta^{a}(n)\left\{\bar{\Psi}(n) \gamma_{5} T^{a} \Psi(n)\right. \\
& \left.+\frac{1}{8} \sum_{\mu}\left[(\bar{\Psi}(n-\hat{\mu})+\bar{\Psi}(n+\hat{\mu})) L T^{a} \Psi(n)+\text { h.c. }\right]\right\}
\end{aligned}
$$

where only the Wilson terms have contributed to the non-invariance of the action, and $\langle. .\rangle_{u}$ denotes the fermionic expectation in the background of the gauge field.

Naively the breakage of gauge invariance is only of order $f^{2}$. This is because the Wilson term corresponds to a dimension-five interaction suppressed by $f$ for the physical modes, and to a mass of $O(1 / f)$ for the doubler modes, whose effects one might expect to be suppressed by their mass. Furthermore, these interactions are chirality flipping and must therefore come in pairs. This naive expectation is however upset by power-like ultraviolet divergences in $1 / f$ in the one loop computation of (10), which can cancel the $f^{2}$ suppression. Of course these ultraviolet divergent parts are local functionals of the gauge fields and $\omega$, the allowed forms of which can be determined by power-counting, lattice rotational symmetry and invariance under space-time independent gauge transformations. The finite parts of $\delta_{\omega} \Gamma_{0}[u]$ can be non-local in $u_{\mu}$, but they are suppressed by the power of $f^{2}$ just discussed. Thus,

$$
\delta_{\omega} \Gamma_{0}[u]=\sum_{n} L(n)+O\left(f^{2}\right)
$$

where $L(n)$ is a local function of $\omega$ and $u_{\mu} . L(n)$ can contain local operators up to dimension 5 . 
We are now in a position to see the difference between the breakage of gauge invariance caused by a non-invariant regulator and that caused by having an uncancelled gauge anomaly. For example, one of the terms allowed in $L(n)$ is

$$
L_{1}(n)=\frac{\alpha}{f^{4}} \sum_{\mu} \operatorname{Tr}\left[\omega(n) u_{\mu}(n) \omega^{-1}(n+\hat{\mu})-u_{\mu}(n)\right]+\text { h.c. }
$$

It is invariant under lattice rotations and it vanishes for constant $\omega$. The power of $1 / f$ is dictated by dimensional analysis since $f$ is the only scale in $\Gamma_{0}$. The coefficient $\alpha$ however, must be extracted from a one-loop lattice diagram. Although the breakage of gauge invariance given by this term is large, the fact that it is local means we can substract it from $\Gamma_{0}$ if there exist a local functional of $u_{\mu}$, $S_{\text {c.t. }}[u]$ such that $\delta_{\omega} S_{c . t .}=L_{1}$. For the present example such a subtraction can be performed,

$$
\begin{aligned}
\Gamma_{r}[u] & =\Gamma_{0}-S_{c . t .}^{1}[u], \\
S_{c . t .}^{1}[u] & =\frac{\alpha}{f^{4}} \sum_{\mu} \operatorname{tr}\left(u_{\mu}(n)-1\right)\left(u_{\mu}^{-1}(n)-1\right),
\end{aligned}
$$

which contains a gauge boson mass counterterm. We are only allowed to subtract local functionals because the non-local part of $\Gamma_{0}$ already correctly reflects the requirements of one-loop unitarity. If a term in $L(n)$ can be cancelled by subtraction of a local functional up to $O\left(f^{2}\right)$ we will refer to it as 'locally integrable'.

In the absence of gauge anomalies, all the possible terms in $L(n)$ are locally integrable. This result for the $\chi w$ action was first proved in [9] at one loop. The full set of gauge field counterterms are ? $^{\text {, }}$

- Mass Counterterms:

$$
\mu_{W}^{2} \frac{1}{f^{2}} \operatorname{Tr}\left[\sum_{\mu}\left(u_{\mu}(n)-1\right)\left(u_{\mu}^{-1}(n)-1\right)\right]
$$

- Wave Function Renormalization:

$$
\frac{1}{f^{4}}\left[\delta Z_{W} \operatorname{Tr} \sum_{\mu \nu}\left(\Delta_{\nu} u_{\mu}\right)^{2}+\delta \zeta \operatorname{Tr}\left(\sum_{\mu} \Delta_{\mu} u_{\mu}\right)^{2}+\delta Z_{N C} \operatorname{Tr} \sum_{\mu}\left(\Delta_{\mu} u_{\mu}\right)^{2}\right]
$$

\footnotetext{
${ }^{\S}$ We follow the notation of ref. [9]. We however do not include the operators involving ghosts since they are not needed in our two-cutoff construction.
} 
- Gauge Boson Self Interaction:

$$
\begin{array}{r}
\delta Z_{3} \frac{1}{f^{4}} \operatorname{Tr} \sum_{\mu, \nu} \Delta_{\mu} u_{\nu}\left[u_{\mu}, u_{\nu}\right], \\
\delta Z_{4, A} \frac{1}{f^{4}} \operatorname{Tr} \sum_{\mu}\left(u_{\mu}-1\right)^{2} \operatorname{Tr} \sum_{\mu}\left(u_{\mu}-1\right)^{2}, \\
\delta Z_{4, B} \frac{1}{f^{4}} \sum_{\mu, \nu} \operatorname{Tr}\left[\left(u_{\mu}-1\right)\left(u_{\nu}-1\right)\right] \operatorname{Tr}\left[\left(u_{\mu}-1\right)\left(u_{\nu}-1\right)\right], \\
\delta Z_{4, N C} \frac{1}{f^{4}} \sum_{\mu} \operatorname{Tr}\left[\left(u_{\mu}-1\right)^{2}\left(u_{\mu}-1\right)^{2}\right] .
\end{array}
$$

The $\Delta_{\mu}$ are lattice derivatives. So, if $L(n)$ is locally integrable, a one-loop subtraction yields $\delta_{\omega} \Gamma_{r}[u]=O\left(f^{2}\right)$ and, despite the lack of a gauge invariant regulator, gauge invariance is recovered in the continuum limit $f \rightarrow 0$, at least at one loop.

The only term that can occur in $L(n)$ which is not locally integrable is the consistent anomaly,

$$
L^{a}(n)=-\frac{i}{12 \pi^{2}} \operatorname{Tr} \theta(n) \epsilon_{\alpha \beta \gamma \delta} \Delta_{\alpha}\left[a_{\beta} \Delta_{\gamma} a_{\delta}-\frac{i}{2} a_{\beta} a_{\gamma} a_{\delta}\right]+O\left(f^{2}\right),
$$

where $u_{\mu}(n)=e^{i f a_{\mu}(n)}, \omega(n)=e^{i \theta(n)}$. A brief description of the derivation of this expression on the lattice is given in section 6 . The gauge color trace is proportional to the anomaly coefficient, $d_{a b c} \equiv \frac{1}{2} \operatorname{Tr}\left[T^{a}\left\{T^{b}, T^{c}\right\}\right]$, of the fermion representations. If it is not zero, the anomaly breaks gauge invariance and cannot be subtracted away with any local counterterms. We will assume that all gauge anomalies cancel. Hence $\Gamma_{r}[u]$ is gauge invariant up to $O\left(f^{2}\right)$.

Having dealt with gauge invariance (at one loop), let us consider the physics that the fermion effective action incorporates. We have used the freedom one has in regulating fields in the ultraviolet to impose two cutoffs on the theory. The physics at distances $\gg b \gg f$ which survive the continuum limit will be insensitive to whether we use one or two cutoffs. In principle we could first integrate out all fermion momenta above $1 / b$ and get a local theory with just one momentum cutoff. It would contain extra higher dimension interactions dependent on $b$ and $f$, some of which would break gauge invariance. But this breakage would be cancelled by the noninvariance of the cutoff procedure to yield just an $O\left(f^{2}\right)$ breaking of gauge invariance in the one-loop amplitudes of the theory. 
This brings us to an important point. We have just seen that all unsuppressed gauge non-invariance at one loop can be subtracted away (when gauge anomalies cancel). What remains of the gauge non-invariance is insensitive to the $f \rightarrow 0$ limit. But there will be one gauge invariant interaction induced by integrating out fermion momenta between $1 / f$ and $1 / b$, which will be sensitive to $f \rightarrow 0$. This is the gauge field kinetic term which must be renormalized by integrating out fermion momenta between $1 / f$ and $1 / b$. The result for its coefficient is,

$$
1 / g_{b}^{2}=1 / g_{0}^{2}+\log (b / f) \frac{t_{2}(F)}{12 \pi^{2}}
$$

where $t_{2}(F)$ is the sum of quadratic Casimir invariants for the fermion representations. Recall that $1 / g_{0}^{2}$ is the original gauge coupling of the two-cutoff theory, multiplying the gauge kinetic term, $S_{g}$. The second term on the right-hand side is just the one-loop renormalization induced by integrating out fermion momenta between the two cutoffs. Therefore $g_{b}$ is to be identified as the single true coupling of the effective one-cutoff theory which must be scaled to zero in the continuum limit $b \rightarrow 0$. In terms of $g_{b}$, the action of the effective one-cutoff theory is insensitive to $f \rightarrow 0$ (assuming, as we have up till now, that our interpolation procedure does not reintroduce sensitivity to $1 / f)$. Having identified the correct physics within the effective one-cutoff theory, we can proceed to always use the explicitly constructed two-cutoff theory for practical purposes.

In a straightforward one-cutoff theory with cutoff $1 / f$, the one-loop suppression of gauge invariance breaking by $f^{2}$ would not have bought us much. The problem is that the real suppression factor is $f^{2} \times$ gauge boson momenta ${ }^{2}$. Once $\Gamma_{r}[u]$ is put into gauge boson loops, gauge boson momenta of order $1 / f$ could arise, cancelling the $f^{2}$ suppression and leading to large violations of gauge invariance. This is not the case in a two-cutoff scheme such as ours. In loops, the gauge boson momenta are of order $1 / b$ at most since they live on the $b$-lattice links, so that deviations from gauge invariance will be suppressed by $O\left(f^{2} / b^{2}\right)$, so long as the interpolation procedure is smooth enough to not reintroduce substantial $O(1 / f)$ momentum components into the interpolated gauge field $u$ (ie. the $b$-lattice softly cuts off the $u_{\mu}$ momenta above $\left.1 / b\right)$. We can then choose $f / b$ small enough for any given precision and space-time volume we wish to work with. Unfortunately, we will show in section 4 that the interpolation procedure of section 3 is not smooth enough for the $\chi w$ scheme outlined here to work! We are therefore led in the next subsection to a scheme which is a simple hybrid of our $\chi w$ 
scheme and the standard Wilson (sw) scheme for vector-like gauge theories. We will show that our interpolation procedure is smooth enough for this improved hybrid scheme to work. It is also quite possible, that a smoother interpolation scheme can be found, in which case the $\chi w$ scheme would be feasible.

Finally, we define the fermion propagator in an external gauge field, $G[u]$, needed in order to define Greens functions with external fermion lines,

$$
G[u]_{n_{1} n_{2}}^{-1}=\frac{\delta}{\delta \Psi\left(n_{1}\right)} \frac{\delta}{\delta \bar{\Psi}\left(n_{2}\right)} S_{f}^{\chi w}[\Psi] .
$$

If we only consider processes without external sterile fermions, then we must have an even number of chirality flips from the Wilson term in the propagator. Since it is the only term breaking gauge invariance and we are not yet integrating over gauge loops, it is easy to see that the propagator is gauge covariant up to order $f^{2}$.

\subsection{Hybrid Regulator}

We describe an alternative lattice construction of the one-loop fermionic effective action which separately regulates the real and imaginary parts. It will automatically be gauge invariant in the limit $f \rightarrow 0$, without subtractions, precisely when gauge anomalies cancel among the fermion representations. This type of separation was introduced in ref.[15] and further discussed in the lattice context in refs. 沺 16. Since the trick used may be unfamiliar we end the section with a brief discussion of the legitimacy of the regulator in preserving the unitarity property of the unregulated fermion determinant.

The basic, well-known observation is that formally the magnitude of the fermion determinant, $e^{\operatorname{Re} \Gamma}$, is parity-even and is just the square root of a vector-like determinant, which we know how to formulate with exact gauge invariance on the lattice. The phase of the chiral fermion determinant, $\operatorname{Im} \Gamma$, is parity-odd but can be regulated by means similar to those of the previous section, though of course this leads to some breakage of gauge invariance. The advantage here is that this breakage is small. As we saw in the last section, it is given by the sum of local dimension $\leq 4$ terms in the gauge field plus non-local terms which are however suppressed by powers of $f^{2}$. It is easy to see from the possible local terms listed in the last section that the only parity-odd term is the consistent anomaly. For anomaly-free theories the breakage of gauge invariance is thus of order $f^{2}$. 
To effect the separation into $\operatorname{Re} \Gamma$ and $\operatorname{Im} \Gamma$, we note that formally,

$$
i \hat{D}=\left[i \hat{D}(i \hat{D})^{\dagger-1}(i \hat{D})^{\dagger} i \hat{D}\right]^{\frac{1}{2}}
$$

Taking the determinant of both sides of the above equation yields

$$
\operatorname{det}[i \hat{D}]=\left\{\operatorname{det}[i \hat{D}] \frac{1}{\operatorname{det}[i \hat{D}]^{*}} \operatorname{det}\left[(i \hat{D})^{\dagger}\right] \operatorname{det}[i \hat{D}]\right\}^{\frac{1}{2}}
$$

Now, since $(i \hat{D})^{\dagger}=i \not D R+i \not \partial L$, while $i \hat{D}=i \not D L+i \not \partial R$, the third and fourth determinant factors in the square root together describe a Dirac fermion with vector-like gauge couplings, and a sterile Dirac fermion. Thus we have,

$$
\operatorname{det}[i \hat{D}]=\left\{\frac{\operatorname{det}[i \hat{D}]}{\operatorname{det}[i \hat{D})]^{*}} \operatorname{det}[i \not D] \operatorname{det}[i \not \partial]\right\}^{\frac{1}{2}}
$$

The last, gauge field independent determinant only contributes a constant to the fermion effective action and can be dropped. The first ratio is a phase factor, while $\operatorname{det}[i \not D]$ is a real, vector-like determinant. Its square-root can consistently be taken as positive as long as the theory does not suffer from Witten anomalies [17]. The idea of this section is to lattice-regulate these determinants separately, in a way which manifestly respects the even and odd parities of $\operatorname{Re} \Gamma$ and $\operatorname{Im} \Gamma$ respectively.

We now have at our disposal a simple means of formulating our new improved chiral fermion determinant,

$$
\operatorname{det}[i \hat{D}]_{h y b r i d} \equiv\left(\frac{\operatorname{det}[i \hat{D}]_{\chi w}}{\operatorname{det}[i \hat{D}]_{\chi w}^{*}}\right)^{\frac{1}{2}} \cdot\left(\operatorname{det}[i \not D]_{s w}\right)^{\frac{1}{2}} .
$$

Here, $\operatorname{det}[i \hat{D}]_{\chi w}$ is just the $f$-lattice chiral determinant of the last section, while $\operatorname{det}[i \not D]_{s w}$ is the standard gauge-invariant Wilson determinant for vector-like fermions. The only difference with single-lattice vector-like theories is that a mass counterterm is still not required, because the mass induced by breaking chiral symmetry via the standard Wilson term is at most $O\left(f / b^{2}\right)$.

As explained earlier in this section, the only breakage of gauge invariance in $\operatorname{det}[i \hat{D}]_{\text {hybrid }}$ must be parity-odd, and therefore suppressed by $f^{2}$ in anomaly free models.

Let us view the above decomposition and regularization in diagrammatic terms. The loop diagrams representing the chiral fermion effective action 
have vertices containing $L=\left(1-\gamma_{5}\right) / 2$. Even before regulating the diagrams one can separate the real (parity-even) and imaginary (parity-odd) parts of the original diagrams. It is easy to check that the diagrams representing (the unregulated) $\operatorname{det}[i \not D]^{1 / 2}$ and $\left(\operatorname{det}[i \hat{D}] / \operatorname{det}[i \hat{D}]^{*}\right)^{1 / 2}$ reproduce exactly these parts respectively. By regulating these determinants separately we are just regulating the parity-even and odd diagrams separately.

This scheme does not harm the unitarity properties possessed by the unregulated diagrams. The point is that any diagram can be written as a non-local part which is insensitive to the cutoff and a local part (polynomial in external momenta) which may be sensitive to the cutoff. Unitarity constrains only the non-local parts, the local terms corresponding to arbitrary 'subtraction constants'.

If composite operators need to be defined, they may require new counterterms in order to recover gauge invariance. See section 6 for the example of global currents.

Finally, amplitudes which involve external fermion lines can continue to be treated using the $\chi w$ propagator in the background of the gauge field.

\section{Gauge Field Interpolation}

We describe our adaptation of a recent suggestion by 't Hooft [3] for interpolating a gauge field living on the links of the $b$-lattice. We consider the two cases of interpolation to any $f$-lattice subdivision of the $b$-lattice, or to the continuum. The latter will be of use since many of the limiting properties of the $f$-lattice interpolation as $f \rightarrow 0$ will be those of the continuum interpolation. After having defined the interpolation algorithm we discuss the important properties that it satisfies. An alternative interpolation which may be computationally simpler is discussed in section 8 .

Broadly speaking, the interpolation is obtained by pasting together solutions of the continuum or $f$-lattice Yang-Mills equations in each fourdimensional hypercube of the $b$-lattice, where the gauge field on the boundaries of the hypercube are specified in the directions tangential to the boundary. This should determine a unique gauge field in the hypercube up to gauge transformations in its interior, which we fix with a Lorentz gauge condition."T The tangential gauge fields on the three-dimensional boundary cubes

\footnotetext{
IThe existence of solutions and their uniqueness once the Lorentz gauge condition is imposed can be proven to all orders in the gauge field self-coupling. This result should apply when the flux through any $b$-plaquette is sufficiently small, as will be the case near
} 
are themselves determined by three-dimensional Yang-Mills equations, and similarly for their own boundaries, the two-dimensional plaquettes. The continuum or $f$-lattice gauge fields tangential to the links of the $b$-lattice are fixed to be constant along the link, and these set the ultimate boundary conditions for the multi-stage interpolation procedure. The details now follow.

Let $U_{\mu}(s)=\exp \left(i b A_{\mu}(s)\right)$ be the gauge field configuration on the $b$ lattice. We can describe the interpolation to either the continuum or the $f$-lattice in terms of a field $a_{\mu}(x)$, which represents the continuum gauge field, or the $f$-lattice gauge field via $u_{\mu}(x)=\exp \left(i f a_{\mu}(x)\right)$ (in which case $x$ is taken to be restricted to the $f$-lattice vertices). First, on points lying on the links of the $b$-lattice we interpolate the tangential gauge field component by,

$$
a_{\mu}(x=s+b \lambda \hat{\mu})=A_{\mu}(s),
$$

where $0 \leq \lambda<1$. The interpolation into a 1-2-directed $b$-lattice plaquette is given by minimizing the two-dimensional Yang-Mills action

$$
S=\int d x_{1} d x_{2} f_{12}^{2}(x)
$$

or

$$
S=\sum_{x} \operatorname{Tr}\left[1-u_{1}(x) u_{2}(x+f \hat{1}) u_{1}^{-1}(x+f \hat{1}+f \hat{2}) u_{2}^{-1}(x+f \hat{2})\right]+\text { h.c. },
$$

in the gauge $\partial_{1} a_{1}+\partial_{2} a_{2}=0$, subject to the boundary conditions given by agreement with the $a_{1}$ and $a_{2}$ already determined on the bordering 1and 2-directed links respectively. The $x$ being integrated (summed) over are restricted to the points inside the $b$-lattice plaquette. $f_{\mu \nu}$ denotes the field strength made from $a_{\mu}$. Note that only the $a_{\mu}$ components tangential to the $b$-plaquette have been determined. The generalization to interpolating in $b$-plaquettes pointing in other directions is obvious.

The way to complete the interpolation procedure is also clear. We interpolate to the interior of any $b$-lattice 3 -cube by minimizing the appropriate three-dimensional Yang-Mills action in three-dimensional Lorentz gauge with the boundary condition being agreement with the tangential gauge field on the plaquettes bordering the cube, determined at the previous stage. This will determine the three components of the gauge field tangential to the cube. Finally we fully interpolate the gauge field to every four-dimensional

the continuum limit. 
hypercube by minimizing the four-dimensional Yang-Mills action in Lorentz gauge with the boundary conditions being agreement with the tangential gauge fields in the 3 -cubes bordering the hypercube. This completes the determination of $a_{\mu}(x)$.

This interpolation procedure is a finite computation (in finite volume) when going from a $b$ - to $f$-lattice. It satisfies several important properties which we now list and briefly discuss.

- Transverse continuity: This describes how smooth our interpolation is. As one passes from one hypercube of the b-lattice into another, the components transverse to the direction taken (ie. tangential to the 3-dimensional boundary) vary continuously because these components were fixed on the boundary as part of the boundary conditions for interpolation into each of the adjacent hypercubes. However the 'longitudinal' component of the gauge field can vary discontinuously. This is also true at lower dimensional levels: passing between adjacent 3-cubes or adjacent plaquettes or adjacent links of the $b$-lattice.

One may wonder what the relevance of transverse continuity is. The point is that the interpolation procedure does not sharply cut off the momenta of the $a_{\mu}$ fields above $\pi / b$ even though of course the $b$-lattice fields we are interpolating have sharply cut off momenta. Rather, the interpolation procedure should be seen as a 'soft' cutoff of the $a_{\mu}$ momenta above roughly $1 / b$. The less smooth the interpolation, the less sharp the cutoff of $a_{\mu}$ momentum. Now suppose $\Gamma[a]$ is a well-defined functional of continuum gauge fields $a_{\mu}$. We will want $\Gamma[a[U]]$ to be a finite functional of $U$. This will fail unless $a[U]$ momentum components fall off fast enough in the ultraviolet. (In an $f$-lattice we will not get an infinity but will get unsubtractable dependences on $1 / f$ which will preclude our taking the $f \rightarrow 0$ limit.) This important consideration is taken up in detail in the next section where it is shown that transverse continuity is sufficient to ensure a well-behaved fermion determinant as a functional of $U_{\mu}$.

- Gauge Invariance: A gauge invariant functional of the interpolated gauge field, $\Gamma[a]$, automatically yields a gauge invariant functional of the $b$-lattice gauge field, $\Gamma[a[U]$. Clearly, it is sufficient to show that

$$
a\left[U^{\Omega}\right]=a^{\omega}[U]
$$

where $\Omega(s)$ is a $b$-lattice gauge transformation of $U_{\mu}$, and $\omega[U, \Omega](x)$ is a continuum ( $f$-lattice) gauge transformation which interpolates $\Omega$ and can depend on $U$. We show that such an $\omega$ exists by describing its construction in the links, then plaquettes, cubes and hypercubes spanned by the $b$-lattice. 
For points on a link of the $b$-lattice in the $\mu$ direction we take

$$
\omega(x=s+\lambda \hat{\mu} b)=\left(\Omega(s) U_{\mu}(s) \Omega^{-1}(s+\hat{\mu} b)\right)^{-\lambda} \Omega(s) U_{\mu}(s)^{\lambda} .
$$

This has the property that $\left.a_{\mu}^{\omega}(s+\lambda \hat{\mu} b)\right)=A^{\Omega}(s)$, where $U_{\mu}^{\Omega}=e^{i A_{\mu}^{\Omega} \text {, which }}$ is indeed the behavior of $a\left[U^{\Omega}\right]$ on points on the $b$-link. Inside a 1-2 directed $b$-lattice plaquette we extend $\omega$ defined on the bounding links in a continuous fashion, so that $a^{\omega}$ is now defined in the plaquette. Since one is always free to take a gauge field to Lorentz gauge via a gauge transformation, $\omega$ can be chosen so that $a^{\omega}$ is in the two-dimensional Lorentz gauge $\partial_{1} a_{1}^{\omega}+\partial_{2}^{\omega} a_{2}=0$. By the gauge invariance of the two-dimensional Yang-Mills action, $a^{\omega}$ yields the minimum action of gauge fields agreeing with the tangential components $a^{\omega}=A^{\Omega}$ already defined on the bounding links. These are precisely the defining properties of the interpolation $a\left[U^{\Omega}\right]$ on the plaquette, so $a^{\omega}=$ $a\left[U^{\Omega}\right]$ there. Again, the remainder of the construction should be clear; $\omega$ can be extended into cubes and hypercubes till one has a gauge transformation everywhere and $a^{\omega}$ satisfies the defining properties of $a\left[U^{\Omega}\right]$.

- Locality: When the momentum space Feynman vertex for the coupling of the interpolation $a_{\mu}$ to the fermions is expressed in terms of $A_{\mu}$, the result is a series of interaction vertices which are polynomial in the $A_{\mu}$ momenta and fields, with higher dimension interactions balanced by powers of $b$. For low momenta $q \ll 1 / b$,

$$
a_{\mu}(q) \sim A_{\mu}(q)
$$

This allows us to view the whole interpolation procedure as an intermediate step in coupling $b$-lattice gauge fields to fermions that live in the continuum ( $f$-lattice) in such a way that the low-energy physics far below $1 / b$ is unaffected. The complexity of the procedure arises from the need to maintain gauge invariance.

The reason locality is satisfied by the interpolation is quite easy to see: $a_{\rho}$ at the point $x=s+y b$ is determined purely by $A_{\mu}(s), A_{\mu}(s+\hat{\nu} b)$, that is by $A_{\mu}(s), \partial_{\nu} A_{\mu}(s)$, and agrees with $A_{\rho}$ on the original links of the $b$-lattice. For low momentum this corresponds to eq.(29). Overall momentum conservation is violated by multiples of $2 \pi / b$ as is familiar in lattice theories in general, the difference in the present context being that the fermion momenta live in the $f$-lattice Brillouin zone not the $b$-lattice Brillouin zone. This is not a problem for locality but is related to taking the $f \rightarrow 0$ limit, which is dealt with in the next section.

- Rotational and translational symmetry: Our interpolation scheme is manifestly invariant under rotations and translations of the $b$-lattice so any 
invariant functional of $a_{\mu}$ translates into an invariant functional of $U_{\mu}$.

- Topological Winding Number: As noted by 't Hooft [3], this type of interpolation allows us to ascribe a winding number to any $b$-lattice configuration whose field strength dies off at infinity, namely the winding number of its continuum interpolation. This will be useful in section 7 , dealing with fermion number violation. Clearly, 'instantons' smaller than $b$ cannot occur as the interpolation of a $b$-lattice gauge field. The basic idea of assigning winding number to lattice gauge fields was first explored in ref. [18].

It is good to see the simplest concrete example of many of the above considerations. This is provided by the interpolation of $U(1)$ gauge fields, for which we can explicitely construct the solution,

$$
a_{1}(x=s+y b)=\sum_{z_{2}, z_{3}, z_{4}=0,1} A_{1}\left(s+b \sum_{\rho \neq 1} z_{\rho} \hat{\rho}\right) \prod_{\nu \neq 1}\left(z_{\nu}\left(2 y_{\nu}-1\right)+1-y_{\nu}\right),
$$

the other components obtained by cyclic permutation. It is easily checked that this interpolation satisfies all the right boundary conditions, is in Lorentz gauge and satisfies Maxwell's equations (minimizes the Maxwell action for the appropriate dimension) in the interiors of hypercubes, 3-cubes and plaquettes, and is transversely but not longitudinally continuous. Also, $b$-lattice gauge fields which are gauge equivalent interpolate to gauge equivalent fields in the continuum or $f$-lattice.

It is also instructive to examine the interpolation in momentum space,

$$
a_{\mu}(q)=A_{\mu}(\bar{q}) \frac{e^{i b \bar{q}_{\mu}}-1}{i\left(b \bar{q}_{\mu}+2 \pi m_{\mu}\right)} \prod_{\nu \neq \mu} \frac{2-2 \cos b \bar{q}_{\nu}}{\left(b \bar{q}_{\nu}+2 \pi m_{\nu}\right)^{2}}
$$

where we decompose continuum ( $f$-lattice) momenta according to

$$
q_{\mu}=\bar{q}_{\mu}+\frac{2 \pi}{b} m_{\mu} \quad \text { where } \quad\left|\bar{q}_{\mu}\right|<\pi / b, \quad \text { and } m_{\mu} \text { is an integer. }
$$

We see that $a_{\mu}$ has momentum components above $\pi / b\left(m_{\mu} \neq 0\right)$, though $A_{\mu}$ does not. But because of the transverse continuity of the interpolation, above $\pi / b$ the momentum components fall off (with $m_{\mu}$ ), faster in the transverse directions than in the longitudinal direction. This type of falloff will be sufficient for our purposes in the next section. At low momentum, $q_{\nu} b \ll 1$, the interpolation looks very simple, $a_{\mu}(q) \sim A_{\mu}(q)$. Finally, note that the interpolation can be expanded as a power series in $b \bar{q}_{\mu}$ so that there is no non-locality introduced by substituting the interpolation into a local interaction vertex of $a_{\mu}(q)$ to fermion fields. 


\section{The $f \rightarrow 0$ Limit}

We have seen in section 2 that $\Gamma_{r}[u]$ at one loop is finite in the limit $f \rightarrow 0$, and gauge invariant up to $O\left(f^{2}\right)$. Also, it was shown that $G[u]$ is gauge covariant up to $O\left(f^{2}\right)$. However, when $\Gamma_{r}$ and $G$ are considered as functionals of the real dynamical variables, $U$, since the interpolation $u[U]$ depends on $f$, we must take care that no extra powers of $1 / f$ are generated. The objective of this section is then to prove,

$$
\lim _{f \rightarrow 0} \quad \Gamma_{r}[u[U]]<\infty, \quad \lim _{f \rightarrow 0} \quad G[u[U]]<\infty
$$

and

$$
\begin{aligned}
\Gamma_{r}\left[u\left[U^{\Omega}\right]\right]-\Gamma_{r}[u[U]] & =O\left(f^{2}\right), \\
G^{\omega^{-1}}\left[u\left[U^{\Omega}\right]\right]-G[u[U]] & =O\left(f^{2}\right),
\end{aligned}
$$

where $\Omega(s)$ is a gauge transformation on the $b$-lattice, while $\omega[U, \Omega]$ is its interpolation to the $f$-lattice. (Recall that our interpolation has the property that $\left.u\left[U^{\Omega}\right]=u^{\omega}[U].\right) \quad G^{\omega^{-1}}$ is the obvious gauge transformed fermion propagator.

\subsection{Fermionic Effective Action}

In perturbation theory, the renormalized one loop effective action in terms of the interpolated fields is given by,

$\Gamma_{r}[u]=\sum_{n} \int_{B Z(f)} \prod_{i} d^{4} q_{i} \Gamma_{\alpha_{1} \alpha_{2} \ldots}^{(n)}\left(q_{1}, . ., q_{n} ; f\right) a_{\alpha_{1}}\left(q_{1}\right) \ldots a_{\alpha_{n}}\left(q_{n}\right) \delta^{(4)}\left(\sum_{i} q_{i}\right)(35$

which can be rewritten as,

$\Gamma_{r}=\sum_{n} \int_{B Z(b)} \prod_{i} d^{4} \bar{q}_{i} \sum_{m_{\mu_{i}}=-b / f}^{b / f} \Gamma_{\alpha_{1} \alpha_{2} \ldots}^{(n)}\left(q_{1}, . ., q_{n} ; f\right) a_{\alpha_{1}}\left(q_{1}\right) \ldots a_{\alpha_{n}}\left(q_{n}\right) \delta^{(4)}\left(\sum_{i} q_{i}\right)(36$

where $q_{i}=\bar{q}_{i}+2 \pi m_{i} / b$ and $m_{\mu_{i}}=-b / f, . ., b / f$.

Although the amplitudes $\Gamma^{(n)}$ are finite in the limit $f \rightarrow 0$, it is clear from eq. (36) that the sums over integers $m_{\mu_{i}}$ could introduce powers of $1 / f$. Obviously, for a smooth enough interpolation, the high momentum components of the interpolated fields are expected to be cut off by $\sim 1 / b$, in such a way that the sums over $m_{\mu_{i}}$ are convergent in the limit $f \rightarrow 0$. 
We now prove that this is indeed the case. For the proof we only need some smoothness properties of the interpolation and a bound on one loop (fermion) lattice integrals for large external momenta $q \sim 1 / f$.

In the previous section we constructed a tranversely continuous interpolation. The gauge field however jumps in the longitudinal direction at the boundaries of the $b$-cell, i.e. $a_{\mu}(n)$ is not continuous in the $\mu$ direction, but it is in any tranverse direction $\nu \neq \mu$. Also, within each $b$-cell it is fully continuous with continuous derivatives to arbitrary order. By continuity on the lattice we mean the following property,

$$
a_{\mu}(n)-a_{\mu}(n-1)=O(f) .
$$

Note that this definition already implies the existence of a derivative that maybe discontinuous, but is bounded for small $f$. For an interpolated field with these properties, it is easy to derive a bound on the high momentum components,

$$
\left|a_{\mu}(q)\right|<C \frac{1}{\left|\hat{q}_{\mu}\right|} \prod_{\alpha \neq \mu} \frac{1}{\left|\hat{q}_{\alpha}^{2}\right|}<C^{\prime} \frac{1}{\left|q_{\mu}\right|} \prod_{\alpha \neq \mu} \frac{1}{q_{\alpha}^{2}},
$$

with

$$
\hat{q}_{\mu} \equiv \frac{e^{i q_{\mu} f}-1}{f}
$$

where $C, C^{\prime}$ are constants independent of $f$. Note that this bound is satisfied by the $U(1)$ interpolation of the previous section.

We sketch the proof for a function of one variable, the extension being straightforward. A function, $F(n)$, defined on the points $n f$, which is not continuous at the boundaries $n f=s b$ (but continuous with continuous derivatives elsewhere) can always be written as the sum of a step-like function $S$ (i.e. constant in each interval $(s-1) b \leq n f<s b)$ plus a continuous function $\tilde{F}$,

$$
F(n)=\tilde{F}(n)+S\left(s \frac{b}{f}\right),
$$

with $n f=s b+\delta n f$, and $0 \leq \delta n<b / f$. The momentum components of $S$ behave as $O(1 /|\hat{q}|)<C /|q|$, for $q \sim 1 / f$. On the other hand, since $\tilde{F}$ is continuous in the sense of (37), its first lattice derivative is bounded in the 
limit $f \rightarrow 0$. Also the momentum components of this derivative are bounded and we can write,

$$
|\tilde{F}(q)|=\left|\frac{(\hat{\partial} \tilde{F})(q)}{\hat{q}}\right|<C \frac{1}{|q|}
$$

This implies that $|F(q)|<C /|q|$, with $C$ independent of $f$. It is easy to repeat the analysis for a continuous function, by writing its derivative as the sum of a step-like function and a continuous function. We find in this case $|F(q)|<C /|q|^{2}$.

The second ingredient in the proof of finiteness is a simplified version of the power counting theorem of Reisz [19]. We use the derivation of reference [20] for one loop diagrams. Here, we simply state the result and refer to appendix A for the details. The renormalized lattice integrals contributing to the $\Gamma^{(n)}$ of (36) satisfy the bound

$$
\left|\Gamma^{(n)}(q ; f)\right|<C|q|^{4-n} \operatorname{Logs}(|q|),
$$

for all $f<f_{0}$ for some fixed $f_{0}$, with $C$ a constant independent of $f$. $n$ is the number of external boson lines and $q$ denotes generically the external momenta. Note that this is also the result expected from naive power counting in the continuum.

Now, we are ready to prove the finiteness of (36). First we note that for $n \geq 4$, eqs. (38) and (42) imply that the sums over $m_{\mu}$ are absolutely convergent in the limit $f \rightarrow 0$. Since the genuine logarithmic divergence in the $n=4$ amplitude has been absorbed into the definition of $g_{b}$ (see eq.(18)), this means that all the contributions with $n \geq 4$ are finite in this limit.

To prove finiteness in the $n=2$ and $n=3$ cases, we have to rely on the transverse properties of these amplitudes for the hybrid action. The P-even contributions are gauge invariant. This implies,

$$
\sum_{\mu} \hat{q}_{\mu} \Gamma_{\mu \nu}^{(2)}(q)=0 \quad \sum_{\mu} \hat{q}_{1,2}^{\mu} \Gamma_{\mu \nu \alpha}^{(3)}\left(q_{1}, q_{2}\right)=C \Gamma_{\nu \alpha}^{(2)}\left(q_{2,1}\right) .
$$

It is shown in Appendix A that these relations are enough to prove the stricter bounds,

$$
\left|\Gamma_{\mu \mu}^{(2)}(q)\right|<C\left|q_{\alpha}\right|^{2} \log (|q|) \quad\left|\Gamma_{\mu \mu \mu}^{(3)}(q)\right|<C\left|q_{\alpha}\right| \log (|q|) \quad \alpha \neq \mu .
$$

Together with (38) and (42), it is now straightforward to prove absolute convergence of the $m_{\mu}$ sums for the parity-even $n=2$ and $n=3$. 
On the other hand, these relations are not satisfied by the $\chi w$ action (7) and in fact we cannot prove convergence of the $m_{\mu}$ sums in (36). This is simple to understand in real space. We have seen that the chiral Wilson action requires a counterterm of the type,

$$
\delta \chi(f) \operatorname{Tr} \sum_{\mu}\left(\Delta_{\mu} u_{\mu}\right)^{2} .
$$

This is not well defined in the $f \rightarrow 0$ limit, because the longitudinal derivative of the interpolated field is a sum of approximate delta functions, whose square is ill-defined. For finite $f$, it would diverge as a power of $b / f$, introducing more divergences than those in $Z_{W}$. In the absence of a smoother interpolation, we conclude that the chiral Wilson action (7) cannot be used.

Finally, we need to consider the parity-odd parts in $n=2,3$. The P-odd amplitude for $n=2$ is exactly zero. On the other hand, the three-point function is not zero. Obviously, since anomalies cancel, these contributions are gauge invariant in the limit $f \rightarrow 0$, but for finite $f$, we cannot use the fact that the diagram is gauge invariant to derive a relation like (44). Nevertheless, this bound still holds because of the presence of an epsilon tensor. It is proven in appendix A by direct inspection. Again this is enough to prove absolute convergence of the $m_{\mu}$ sums.

This concludes the proof that the one-loop renormalized fermionic effective action derived for the hybrid scheme (23) has a good limit $f \rightarrow 0$.

We still need to prove that the gauge non-invariant contributions that remain after renormalization are $O\left(f^{2}\right)$. In section 2 , we have seen that this is true for $\Gamma_{r}[u]$. Here we prove that, when the $f$ dependence of the interpolation is taken into account in $\Gamma_{r}[u[U]]$, no extra powers of $1 / f$ change this result (34). For this we just need to extend the bounds (42) to amplitudes with one insertion of $\delta_{\omega} S_{f}^{\chi w}$. We denote these amplitudes by $\delta_{\omega} \Gamma^{(n)}$. Under a gauge transformation on the $b$-lattice, $\Gamma_{r}$ for the hybrid scheme changes by,

$$
\begin{array}{r}
\delta_{\omega} \Gamma_{r}=\delta_{\omega} \ln \left(\frac{\operatorname{det} i \hat{D}}{\operatorname{det}[i \hat{D}]^{*}}\right)^{\frac{1}{2}}=\operatorname{Im}\left[<\delta_{\omega} S_{f}^{\chi w}>_{\chi w}\right]= \\
\sum_{n} \int_{B Z(b)} \prod_{i} d^{4} \bar{q}_{i} \sum_{m_{\mu_{i}}=-b / f}^{b / f} \delta_{\omega} \Gamma^{(n)}\left(q_{i}\right) a\left(q_{1}\right) a\left(q_{2}\right) \ldots a\left(q_{n}\right) \delta^{(4)}\left(\sum_{i} q_{i}\right),
\end{array}
$$

where, $S_{f}^{\chi w}$ is given by the action ([). In momentum space, we get

$$
\delta_{\omega} S_{f}^{\chi w}=i \theta^{a}(q) \delta^{(4)}\left(q+p-p^{\prime}\right) \bar{\Psi}\left(p^{\prime}\right)\left\{M\left(p^{\prime}\right) L-M(p) R\right\} T^{a} \Psi(p),
$$


for an infinitesimal gauge transformation $\omega=e^{i \theta^{a}(n) T_{a}}$, obtained by interpolating an infinitesimal $b$-lattice gauge transformation $\Omega(s)$ (see section 3 ), and $M(p) \equiv(2 r / f) \sum_{\nu} \sin ^{2}\left(p_{\nu} f / 2\right)$. Inserting this term into a free fermion propagator gives,

$$
-i\left[\frac{M\left(p^{\prime}\right)^{2}}{P_{f}^{2}\left(p^{\prime}\right)} \frac{1}{P_{f}(p)}-\frac{M(p)^{2}}{P_{f}^{2}(p)} \frac{1}{P_{f}\left(p^{\prime}\right)}\right],
$$

where $1 / P_{f}$ is the free fermion propagator. Also, we have the bound,

$$
\left|\frac{1}{f^{2}} \frac{M^{2}(p)}{P_{f}^{2}(p)}\right|<C p^{2},
$$

for all $f<f_{0}$ and $C$ some constant. From the analysis of Appendix A, we conclude that a diagram with one insertion of $\delta_{\omega} S_{f}$ and $n$ gauge legs, is the sum of local terms up to dimension 5 plus a non-local part which is bounded by,

$$
\left|\delta_{\omega} \Gamma^{(n)}\right| \leq f^{2}|q|^{6-n} \operatorname{Logs}(|q|) .
$$

Since there is no local P-odd operator of dimension 5 or less, except the anomaly, which vanishes, the non-local part bounded by $(50)$ is all there is and as expected is $O\left(f^{2}\right)$.

It is clear from the previous discussion that amplitudes with $n \geq 6$ have two or more powers of $f^{2}$ and since for large momentum, $q \sim 1 / f$, they must satisfy bound (50), we can use it together with (38), to prove that the sums over $m_{\mu}$ in (46) are absolutely convergent and do not bring any extra powers of $1 / f$.

For $n \leq 5$, we make use of the fact that there is an epsilon tensor. In the case of $n=2$, it can be shown by direct inspection that the integral is bounded by,

$$
\left|\delta_{\omega} \Gamma_{\mu \nu}^{(2)}(q)\right|<C f^{2}\left|q_{\alpha}\right|\left|q_{\beta}\right||q|^{2} \log (|q|) \quad \alpha, \beta \neq \mu, \nu \quad \alpha \neq \beta
$$

Using (38) and the full continuity of the interpolated gauge transformation $\theta(n)$, which leads to a bound of the form

$$
|\theta(q)|<C \prod_{\alpha} \frac{1}{q_{\alpha}^{2}}
$$

it is easy to see that the sums over $m_{\mu}$ yield a result suppressed by $f^{2}$ up to logs. A similar analysis shows this is true for $n=3-5$ diagrams. This ends the proof of (34). 


\subsection{Fermion Propagator}

We finally need to consider the propagator $G[u[U]]$. We will prove that it has a well-defined $f \rightarrow 0$ limit and that the breakage of gauge invariance is down by at least $O\left(f^{2}\right)$. In perturbation theory, it is given by,

$G[U]\left(q, q^{\prime}\right)=\sum_{n} \int_{B Z(b)} \prod_{i=1}^{n} d \bar{p}_{i} \sum_{m_{\mu_{i}}} G^{(n)}\left(p_{1}, . ., p_{n}\right) a\left(p_{1}\right) \ldots a\left(p_{n}\right) \delta^{(4)}\left(q^{\prime}-q-\sum_{i=1}^{n} p_{i}\right)(53$

where as usual $p_{i}=\bar{p}_{i}+2 \pi m_{i} / b$. Since, the amplitudes $G^{(n)}$ are products of free propagators, and $\left|P_{f}(k)\right|<C /|k|$, this implies that for large $m_{\mu}$, we must have $\left|G^{(n)}\right|<C /\left|p_{i}\right|$, for any large $p_{i}$. This bound together with (38) implies the absolute convergence of the sums over $m_{\mu}$ in (53). The $f \rightarrow 0$ limit of $G[U]$ is well-defined.

Now we have to prove that the gauge non-invariant contributions to these diagrams are $O\left(f^{2}\right)$. The gauge variation of $G[U]$ is given perturbatively by a sum of diagrams with an arbitray number of gauge boson legs and one insertion of $\delta_{\omega} S_{f}^{\chi w}$. Using (47) and (49), we see that the line with the insertion can be bounded for large $m_{\mu}$ by $f^{2}|k|$ where $k$ is the momentum of the fermion line, as compared to the behaviour $\sim 1 /|k|$ without the insertion. From (53), it is clear that the sums over $m_{\mu}$ are at most logarithmically divergent in this case, so that the breakeage of gauge invariance in $G[U]$ is at most $O\left(f^{2} \log (f)\right)$, as expected.

\section{Gauge Ward Identities.}

We are now in a position to demonstrate the gauge invariance of our construction when gauge fields, $U$, are integrated in the functional integral. We have shown in the previous section that the three components of the full functional integral (see eq. (5)), $\Gamma_{r}[u[U]], G[u[U]], S_{g}[U]$, satisfy gauge invariance up to $O\left(f^{2}\right)$ (in fact we showed that mathematically the gauge invariant limit $f \rightarrow 0$ exists, but in any simulation $f$ will be small and nonzero). In the absence of gauge fixing, the full generating functional satisfies,

$$
\begin{aligned}
& \int \prod_{s} d U_{\mu}(s) e^{-S_{g}[U]+\sum_{s} J_{\mu} A_{\mu}^{\Omega}} e^{\Gamma_{r}\left[u\left[U^{\Omega}\right]\right]} e^{-\bar{\eta} G^{\omega^{-1}}\left[u\left[U^{\Omega}\right]\right] \eta}= \\
& \int \prod_{s} d U_{\mu}(s) e^{-S_{g}[U]+\sum_{s} J_{\mu} A_{\mu}} e^{\Gamma_{r}[u[U]]} e^{-\bar{\eta} G[u[U]] \eta}+O\left(\frac{f^{2}}{b^{2}}\right),
\end{aligned}
$$


where $\Omega$ is any $b$-lattice gauge transformation while $\omega[\Omega, U]$ is its $f$-lattice interpolation described in section 3 . The gauge transformed propagator is given by,

$$
G^{\omega^{-1}}\left[u\left[U^{\Omega}\right]\right]\left(n_{1}, n_{2}\right) \equiv \omega^{-1}\left(n_{1}\right) G\left[u\left[U^{\Omega}\right]\right]\left(n_{1}, n_{2}\right) \omega\left(n_{2}\right) .
$$

The central observation needed to understand eq.(54) is that since the $U$ fields live on the $b$-lattice, the largest momentum scale they can introduce when integrated is $O(1 / b)$. That is, they cannot reintroduce any powers of $1 / f$ which could cancel the $f^{2}$ suppression of gauge symmetry violation. Note that this is a non-perturbative expectation $\$$.

For perturbative investigations it is useful to add the standard, rotationally invariant gauge-fixing and ghost terms, neglecting the small breakage of gauge invariance in $\Gamma_{r}[u[U]]$ and $G[u[U]]$ in the Fadeev-Popov procedure. This results in lattice BRST identities among the Green functions which are broken only by $O\left(f^{2} / b^{2}\right)$, since the gauge boson loop momenta are cut off above $O(1 / b)$. The statement of the gauge invariance and unitarity of the quantum theory is contained in the BRST identities. From these it should also be possible to prove perturbative renormalizability and the continuum (renormalized) BRST identities of the theory. Of course, it is believed that there is a unique continuum theory with the particle content of our lattice construction which satisfies the BRST identities pertubatively, and nonperturbatively coincides with the non-gauge-fixed theory.

Note that to compute physical amplitudes, it is essential to be able to form gauge invariant combinations of the external fermion fields and the gauge fields. This, at first sight, seems problematic since the fields live on different lattices, but clearly the solution is to make use of the gauge field interpolation $u[U]$ in forming path-ordered exponentials of the gauge field connecting external fermions.

Let us turn to the question of how small to take the ratio $f / b$ in a lattice simulation in a (physical) volume, $L^{4}$. Clearly we should take $f / b$ small enough that the measurements in our volume are insensitive to the breakage of gauge invariance. For example, a simple perturbative estimate suggests that (in the gauge-fixed theory) the $O\left(f^{2} / b^{2}\right)$ breaking of the Ward identities can induce a gauge boson mass of order $f / b^{2}$. Clearly, if $f / b<b / L$

\footnotetext{
"Small deviations from exact gauge invariance, such as a small bare gauge boson mass term, lead to difficulties in continuum perturbation theory, as is well known. Indeed one is really outside the perturbative regime. However non-perturbatively there is no sign of difficulty. For a brief review of the lattice literature on the example of bare gauge boson masses see ref. 21.
} 
we would be insensitive to this violation of gauge invariance in our volume, since the gauge boson correlation length would be larger than $L$. With such a relationship of scales, in the continuum or thermodynamic limits, $b / L \rightarrow 0$, gauge invariance becomes exact $(f / b \rightarrow 0)$.

\section{Anomalies in Gauge and Global Currents}

In this section we will first check that our hybrid scheme reproduces the consistent gauge anomaly at the one-loop level. As explained in section 2 , this is to be expected on general grounds. Obviously, we only consider theories where the gauge anomaly vanishes (i.e. $d_{a b c}$ among the fermion representations is zero). However, in most theories of interest, there are also other currents associated with global symmetries that can be anomalous (e.g. $\mathrm{B}+\mathrm{L}$ number in the Standard Model). We will show that these are also properly reproduced. In studying current conservation, we will need to consider Green functions with one insertion of the global current. In general, new counterterms are needed to ensure gauge invariance of amplitudes containing composite operators such as this current, even in the hybrid scheme. Once these counterterms have been added, we show that our construction gives the correct gauge invariant anomaly at the one-loop level. It is then straightforward to see that the Adler-Bardeen theorem [22] must hold to all orders in perturbation theory.

The axial anomaly triangle in a vector-like theory was computed in lattice perturbation theory with standard Wilson fermions by several authors [8] 23]. The case we have to consider here is slightly different, since we are in a chiral gauge theory ${ }^{* *}$. The gauge anomaly triangle for our hybrid scheme is given by the imaginary part of the diagram with one insertion of (47) and two gauge boson legs, in the limit $f \rightarrow 0$ (recall that in the hybrid scheme only $\operatorname{Im} \Gamma$ breaks the gauge symmetry). The result we get is,

$$
\begin{array}{r}
\delta_{\omega} \Gamma_{\mu \nu}^{(2)}{ }^{b c}\left(q, q^{\prime}\right)=\frac{i}{\pi^{4}} \theta_{a}\left(q+q^{\prime}\right) d_{a b c} \epsilon_{\mu \alpha \nu \beta} q_{\alpha}^{\prime} q_{\beta} \times \\
\int_{-\pi}^{\pi} d^{4} k c_{\mu} c_{\alpha} c_{\nu} s_{\beta}^{2} \frac{r M s^{2}-c_{\beta} M^{2}}{\left(s^{2}+M^{2}\right)^{4}}+O\left(f^{2}\right)
\end{array}
$$

where $\omega=\exp \left(i \theta^{a} T^{a}\right)$. Also $c_{\alpha} \equiv \cos \left(k_{\alpha}\right), s_{\alpha} \equiv \sin \left(k_{\alpha}\right), M \equiv 2 r \sum_{\alpha} \sin \left(k_{\alpha} / 2\right)^{2}$ and $s^{2} \equiv \sum_{\alpha} \sin \left(k_{\alpha}\right)^{2} . \quad r$ is the Wilson parameter. This integral can be

\footnotetext{
${ }^{* *}$ For a discussion of gauge and global anomalies in the context of the 'overlap' formulation of chiral gauge theories see ref. 24.
} 
solved exactly $\square$ and gives a result independent of $r$ as expected,

$$
\delta_{\omega} \Gamma^{(2)}\left(q, q^{\prime}\right)=\frac{i}{12 \pi^{2}} \theta^{a}\left(q+q^{\prime}\right) d^{a b c} \epsilon_{\mu \alpha \nu \beta} q_{\alpha}^{\prime} q_{\beta}+O\left(f^{2}\right) .
$$

The Wess-Zumino consistency conditions 12 then give the second term of the gauge anomaly,

$$
\delta_{\omega} \Gamma=-\frac{i}{12 \pi^{2}} \epsilon_{\alpha \mu \beta \nu} \operatorname{Tr}\left[\theta \partial_{\alpha}\left(a_{\mu} \partial_{\beta} a_{\nu}-\frac{i}{2} a_{\mu} a_{\beta} a_{\nu}\right)\right]+O\left(f^{2}\right) .
$$

We have proven earlier that, in the absence of gauge anomalies, the hybrid scheme gives a gauge symmetric continuum limit. However, the chiral gauge theories we want to simulate typically contain global $U(1)$ 's which are anomalous. We will consider a general case, in which a global current is denoted by $j_{\mu}$, with generator $t$. We will assume that $t$ commutes with the $T_{a}$. A convenient way to find the anomaly is introducing an external source $b_{\mu}(x)$ that couples to the global current. It also lives on the links of the $f$-lattice: $\tilde{u}_{\mu}=\exp \left(i f b_{\mu} t\right)$. The simplest choice is to couple it like the gauge field (any other choice would lead to the same result). In the hybrid scheme we just substitute,

$$
u_{\mu} \rightarrow u_{\mu} \tilde{u}_{\mu}
$$

both in the chiral Wilson action (7) and in the standard Wilson action, defining $\operatorname{det}[i \hat{D}]_{\chi w}$ and $\operatorname{det}[i \not D]_{s w}$ in $(23)$.

Note that for the chiral Wilson action this prescription corresponds to only the physical left-handed fermion field transforming under the global symmetry.

Due to the presence of the external field $\tilde{u}$, the fermionic effective action is no longer gauge invariant for $d_{a b c}=0$. Instead we get,

$\delta_{\omega} \Gamma[u, \tilde{u}]=-\frac{i}{12 \pi^{2}} \epsilon_{\alpha \mu \beta \nu} \operatorname{Tr}\left[\theta \partial_{\alpha}\left(2 a_{\mu} \partial_{\beta} b_{\nu}-\frac{i}{2} b_{\mu} a_{\beta} a_{\nu}\right)\right]+O\left(b_{\mu}^{2}\right)+O\left(f^{2}\right)(60)$

Since we are only interested in one insertion of the current, we just need the linear terms in $b_{\mu}$. It is easy to see that by adding the local counterterm,

$$
S_{c . t .}[a, b]=\frac{i}{12 \pi^{2}} \epsilon_{\alpha \mu \beta \nu} \operatorname{Tr}\left[2 b_{\alpha} a_{\mu} \partial_{\beta} a_{\nu}-i \frac{3}{2} b_{\alpha} a_{\mu} a_{\beta} a_{\nu}\right]
$$

\footnotetext{
${ }^{\dagger \dagger}$ We thank Ph.Boucaud for pointing this out to us.
} 
one recovers gauge invariance,

$$
\delta_{\omega}\left(\Gamma+S_{\text {c.t. }}\right)=O\left(f^{2}\right) .
$$

Finally, after this subtraction, the global anomaly is given by,

$$
\left.\delta_{\lambda}\left(\Gamma+S_{c . t .}\right)\right|_{b_{\mu}=0}=\left.\operatorname{Im}\left(<\delta_{\lambda} S_{F}^{\chi w}>_{\chi w}\right)\right|_{b_{\mu}=0}+\left.\delta_{\lambda} S_{c . t .}\right|_{b_{\mu}=0}
$$

where $\lambda=e^{i \phi(n) t}$. The first term corresponds to a diagram which is identical to the one for the gauge anomaly, with $\phi t$ replacing $\theta_{a} T_{a}$ in (58),

$$
\left.\delta_{\lambda} \Gamma\right|_{b=0}=-\frac{i}{12 \pi^{2}} \phi \epsilon_{\alpha \mu \beta \nu} \operatorname{Tr}\left[t \partial_{\alpha}\left(a_{\mu} \partial_{\beta} a_{\nu}-\frac{i}{2} a_{\mu} a_{\beta} a_{\nu}\right)\right]+O\left(f^{2}\right)
$$

When we add the counterterm we find,

$$
\left.\phi \partial_{\mu} j_{\mu} \equiv \delta_{\lambda}\left(\Gamma+S_{c t}\right)\right|_{b_{\mu}=0}=-\frac{i}{16 \pi^{2}} \phi \operatorname{Tr}\left[t f_{\mu \nu} \tilde{f}_{\mu \nu}\right]+O\left(f^{2}\right)
$$

with $f_{\mu \nu}$ being the gauge field strength. This is the expected gauge invariant form of the anomaly.

This is the result at one loop. However, since gauge loops can at most generate extra powers of $1 / b$, all the $O\left(f^{2}\right)$ effects in (65) go to zero in the continuum limit, $f / b \rightarrow 0$, to all orders in perturbation theory. This means that in the continuum limit, we recover the well-known result of the Adler-Bardeen theorem since,

$$
<\partial_{\mu} j_{\mu} F>=<-\frac{i}{16 \pi^{2}} \operatorname{Tr}\left[t f_{\mu \nu} \tilde{f}_{\mu \nu}\right] F>+<\delta_{t} F>+O\left(f^{2} / b^{2}\right)
$$

where $F[U, \Psi]$ is an arbitrary functional.

\section{Fermion Number Violating Amplitudes.}

Consider the example of a theory with one generation of standard model fermions interacting with gauge fields but no scalars (since we have not considered them in this paper). In this section we will understand the phenomenon of $B+L$ violation [25] in an 'instanton gas' picture, in the continuum and then on the lattice. This will be sufficient to demonstrate that $B+L$-violating processes will occur in our lattice construction. We then give a prescription for defining lattice-regulated net- $B+L$ violating amplitudes. A similar discussion has been previously given in reference [24], from the point of view of the 'overlap' formulation of chiral gauge theories. 
The main problem is that we have restricted the lattice construction to net fermion number conserving amplitudes (for every external $\Psi_{L}$ we have a corresponding external $\bar{\Psi}_{L}$ ). Still, as we saw in the last section, even in this sector of the theory there is local $B+L$ violation, as given by the anomaly. This will allow us to infer $B+L$ violation in the full theory. The strategy for defining more general correlators, say $\langle\mathcal{O}(x)>$ representing the amplitude for a $B+L$-violating process in the vicinity of $x$, is to demand cluster decompostion of the full theory and consider instead the fermion number conserving correlator [10,

$$
<\mathcal{O}(x) \mathcal{O}^{\dagger}(y)>_{|x-y| \rightarrow \infty} \sim<\mathcal{O}(x)><\mathcal{O}^{\dagger}(y)>,
$$

which is defined in our construction. That is, if this two-point correlator does not vanish as $x$ and $y$ are taken arbitrarily far apart, then $B+L$ is being locally violated (with opposite signs) at $x$ and $y$. Since events at $y$ are too distant to affect those at $x$ in a sensible theory, we must have that

$$
<\mathcal{O}(x)>\neq 0
$$

Inferring fermion number violation by considering fermion number conserving correlators has been exploited in refs. [26].

We will first see how this works formally in the continuum, using the formalism of Fujikawa for chiral theories [27], and then consider our lattice construction afterwards. For simplicity, we will take

$$
\mathcal{O}(x)=q_{L}(x) q_{L}(x) q_{L}(x) \ell_{L}(x)
$$

where we take the internal indices to be contracted gauge invariantly. This operator carries two units of $B+L$. We then have

$$
\mathcal{O}^{\dagger}(y)=\bar{q}_{L}(y) \bar{q}_{L}(y) \bar{q}_{L}(y) \bar{\ell}_{L}(y) .
$$

For a fixed background gauge field $a_{\mu}$, we can decompose the chiral quantum fields in terms of the complete, orthonormal set of eigenfunctions of the vector-like hermitian Dirac operator, $i \not D$,

$$
\begin{aligned}
i \not D \psi_{n} & =\lambda_{n} \psi_{n} \\
\Psi_{L} & =\sum_{n>0} a_{n} \psi_{n}^{L} \\
\bar{\Psi}_{L} & =\sum_{n>0} \bar{a}_{n} \psi_{n}^{R \dagger}
\end{aligned}
$$


Here, we are using a condensed notation where really there are separate Dirac equations for the $\Psi_{L}=q_{L}, \ell_{L}$ fields. The two-point correlator of $\mathcal{O}$ is then given by functional integration over the fermion coefficients $\bar{a}_{n}, a_{n}$,

$$
<\mathcal{O}(x) \mathcal{O}^{\dagger}(y)>_{a_{\mu}}=\left(\prod_{n>0} \lambda_{n}^{q}\right)^{3}\left(\prod_{n>0} \lambda_{n}^{\ell}\right) e^{i \operatorname{Im} \Gamma}\left(\sum_{n>0} \frac{q_{n}^{L}(x) \bar{q}_{n}^{R \dagger}(y)}{\lambda_{n}^{q}}\right)^{3}\left(\sum_{n>0} \frac{\ell_{n}^{L}(x) \bar{\ell}_{n}^{R \dagger}(y)}{\lambda_{n}^{\ell}}\right),
$$

where $\langle\ldots\rangle_{a_{\mu}}$ denotes the fermionic averaging done in the background of $a_{\mu}$, which is presently unintegrated. The origin of the various terms is as follows. The products of eigenvalues for the various fields comes from integrating the fermion action, which is diagonalized in this basis. This is nothing but the square root of the vector-like determinant, which we gaugeinvariantly regulated in section 2 . The phase factor emerges as a Jacobian for a formally unitary transformation,

$$
\int \mathcal{D} \Psi_{L} \mathcal{D} \bar{\Psi}_{L}=\int \prod_{n>0} d a_{n} d \bar{a}_{n} e^{i \operatorname{Im} \Gamma}
$$

The bilinears in the eigenfunctions are the fermion propagators in the eigenbasis.

Now we specialize to the case of a zero winding number background gauge field which is made out of a distantly separated instanton and antiinstanton pair, $a_{\mu}+\tilde{a}_{\mu}$, the instanton in the vicinity of $x$ and the antiinstanton in the vicinity of $y$. To understand the dominant contribution to our two-point correlator we note that for a single instanton, the AtiyahSinger index theorem 28] tells us that each of the fields comprising $\mathcal{O}$ has a left-handed zero mode of its associated vector-like Dirac eigen-equation. For an anti-instanton background, there are right-handed zero modes of the Dirac eigen-equation instead. In the background of the instanton antiinstanton pair, the left and right-handed zero modes pair up to form a Dirac spinor eigenfunction with non-zero eigenvalue, $\lambda_{1}$. As the instanton and anti-instanton are moved far apart, $\lambda_{1} \rightarrow 0$ and the two chiralities separate spatially. As this happens the fermion propagators are dominated by the $\lambda_{1}$ modes,

$$
\sum_{n>0} \frac{\psi_{n}^{L}(x) \bar{\psi}_{n}^{R \dagger}(y)}{\lambda_{n}} \sim \frac{\psi_{1}^{L}(x) \bar{\psi}_{1}^{R \dagger}(y)}{\lambda_{1}}
$$

while the product of eigenvalues is suppressed by $\lambda_{1}$. Cancelling the powers 
of $\lambda_{1}$ gives

$$
<\mathcal{O}(x) \mathcal{O}^{\dagger}(y)>_{a_{\mu}+\tilde{a}_{\mu}} \sim\left(\prod_{n>1} \lambda_{n}^{q}\right)^{3}\left(\prod_{n>1} \lambda_{n}^{\ell}\right) e^{i \operatorname{Im} \Gamma}\left(q_{1}^{L}(x) \bar{q}_{1}^{R \dagger}(y)\right)^{3}\left(\ell_{1}^{L}(x) \bar{\ell}_{1}^{R \dagger}(y)\right)
$$

where the leading behaviors of $\psi_{1}^{L}$ and $\bar{\psi}_{1}^{R \dagger}$ are given by the zero modes of the instanton and anti-instanton respectively.

The mechanism by which $\left\langle\mathcal{O O}^{\dagger}\right\rangle$ survives as $|x-y| \rightarrow \infty$, when we integrate over gauge fields in a dilute instanton gas approximation, is now clear. There will be a purely perturbative contribution to the correlator which will fall to zero as $|x-y| \rightarrow \infty$ and a contribution from an instanton anti-instanton pair of the above form which does not fall off, but rather factorizes into contributions from the vicinities of $x$ and $y$. We are forced to conclude that $B+L$ violation occurs in the full theory. Of course, we expect the qualitative point we are making to survive the (rather drastic) dilute gas approximation. The moral is that the physics of non-trivial winding number is implicit in the zero winding number sector and this is linked to $B+L$ violation through the (integral of the) anomaly equation for the $B+L$ current.

Let us now see how all this relates to our lattice construction. As described in section 3 we mean by a single 'instanton', a $b$-lattice configuration whose interpolation to the continuum yields a unit winding number continuum gauge field. This is easily arranged by obtaining the original $b$-lattice gauge field by discretizing a large (compared with $b$ ) continuum instanton in the first place. An instanton and anti-instanton are then easily composed to give a zero winding number gauge field. For $f \ll b \ll$ instanton size, the hybrid regulator gives essentially the same result as our continuum analysis except that the product of eigenvalues is cut off above roughly $1 / f$. The vector-like determinant is determined by the gauge invariant standard Wilson action. We can work with the related set of lattice eigenfunctions, which for small $f$ we expect to approximate the Fujikawa eigenfunctions of our continuum discussion. The gauge field interpolation procedure makes the remaining analysis as simple as the formal continuum case. The fermion propagators are nearly the same as the continuum propagators in the background of the interpolated gauge field. The poles corresponding to the doubler modes have completely decoupled. Therefore the $\mathcal{O}$ two-point correlator is dominated by the approximate zero modes just as in the earlier analysis. For $|x-y| \rightarrow \infty$ the correlator must be interpreted as $B+L$ violation occurring at $x$ and at $y$ but without any physical connection between 
the two points.

The above discussion leads us to a straightforward means of defining arbitrary lattice-regulated fermion number violating amplitudes in terms of the fermion conserving amplitudes we have already defined. For example, let $\tilde{\mathcal{O}}$ be a product of fields at various points, which carries two units of $B+L$. Then for $x$ far removed from all these points, cluster decomposition yields

$$
<\tilde{\mathcal{O}} \mathcal{O}^{\dagger}(x)>\sim<\tilde{\mathcal{O}}><\mathcal{O}^{\dagger}(x)>.
$$

We can satisfy this by defining

$$
<\tilde{\mathcal{O}}>\equiv \frac{<\tilde{\mathcal{O}} \mathcal{O}^{\dagger}(x)>}{\left(<\mathcal{O}(x) \mathcal{O}^{\dagger}(y)>\right)^{\frac{1}{2}}},
$$

for $y, x$ also distantly separated. Note that all the quantities on the righthand side are defined in the zero-winding number sector ${ }^{\ddagger \ddagger}$.

\section{Computational Efficiency.}

Obviously, the most computationally expensive operation in a simulation of chiral gauge theory using the methods of this paper, compared with lattice QCD say, is the calculation of the fermion determinant on the f-lattice, instead of the coarser $b$-lattice. The other major complication is the gauge field interpolation technique. Let us see what can be done to reduce these costs.

In the hybrid scheme described in section 2 , there are in fact two determinants, the $s w$ and $\chi w$ ones, which must be calculated on the $f$-lattice for each gauge field configuration. The consistent use of the $f$-lattice for fermions was done just to reduce confusion. It is however possible, and much more efficient to calculate the $s w$ determinant directly on the $b$-lattice! The point is that the $s w$ determinant is exactly gauge invariant and we know how to non-perturbatively restore the global chiral symmetry broken by the standard Wilson term on a single lattice for gauge fields and fermions. Just as in lattice QCD, we can use the action

$$
S_{s w}=\frac{b^{3}}{2} \sum_{s, \mu} \bar{\Phi}(s) \gamma_{\mu} U_{\mu} \Phi(s+\hat{\mu})-\bar{\Phi}(s+\hat{\mu}) \gamma_{\mu} U_{\mu}^{-1} \Phi(s)
$$

\footnotetext{
${ }^{\ddagger}$ Actually, there is also an undetermined phase in $\langle\mathcal{O}\rangle$, which we have taken as zero. Its value in general defines the $\theta$-vacuum we occupy.
} 


$$
\begin{aligned}
& +(4 r+M b) b^{3} \sum_{s} \bar{\Phi}(s) \Phi(s) \\
& -\frac{r b^{3}}{2} \sum_{s, \mu}\left[\bar{\Phi}(s) U_{\mu}(s) \Phi(s+\hat{\mu})+\bar{\Phi}(s+\hat{\mu}) U_{\mu}^{-1} \Phi(s)\right],
\end{aligned}
$$

where $M$ has been tuned to the point of chiral symmetry for the vectorlike fermions $\Phi$, by tuning the associated Goldstone bosons to be massless. We see that in this formulation, there is no need to do coupling constant renormalization for distances from $f$ to $b$. That is,

$$
g_{b}=g_{0}
$$

The $\chi w$ determinant which is used to regulate $\operatorname{Im} \Gamma$ must still be regulated on the $f$-lattice because it is not exactly gauge invariant, unlike the $s w$ determinant. Thus the workload has been cut in half, but not more.

Similarly, one can use the $b$-lattice to regulate external fermions. If a $\chi w$ action is used on the $b$-lattice, the breakage of gauge invariance would not be suppressed. But instead, we can use the standard Wilson action above, of course restricting ourselves to calculating with the external fermions carrying the gauge charge.

For conceptual purposes, we have already considered matching the twocutoff theory with a one-cutoff theory. Here we note that such a matching would result in our ability to compute in a chiral theory with the same efficiency as in (unquenched) lattice QCD! The Rome group proposal is similar to this one cutoff theory; they employ the $\chi w$ regulator scheme with gauge fixing terms and demand that BRST relations among the Green functions are obeyed in any simulation, hoping to thereby tune the counterterm coefficients to all orders. However, non-perturbatively the viability of this procedure is unclear. On the other hand, one can imagine computing a handful of Green functions using our two-cutoff method, possibly in a volume not too much larger than $b^{4}$. It may then be possible to tune the counterterms in a single $b$-cutoff theory in order to reproduce the same results to good precision. Once the counterterms are determined in this manner they are fixed for all other possible Green functions. Therefore one can simply proceed to use the single cutoff theory from then on. This corresponds precisely to a non-perturbative matching having been performed at $b$ between the two-cutoff and one-cutoff theories.

Next, we observe that the size of the breakage of gauge invariance is partly determined by the form of the Wilson term in the $\chi w$ regulator. The 
fact that it turned out to be $O\left(f^{2} / b^{2}\right)$ is directly linked to the fact that the $f$ lattice Wilson term possessed two lattice derivatives. But nothing prevents us from using a four-derivative Wilson term, for example. This will push the unsubtractable non-local breakage of gauge invariance to higher order in $f$. If all the lower dimensional local terms are still locally integrable we could arrange to have the Ward identities obeyed to higher precision than $f^{2} / b^{2}$, so a much coarser $f$-lattice could be used. There is a problem however in that the interpolation will almost certainly need to be smoother than it presently is. For example, a four-derivative counterterm would be very singular with $1 / f$ for an interpolation which is only transversely continuous. This kind of improvement will have to await the development of a smoother interpolation and the check of whether all the local terms that might appear in $L(n)$ are locally integrable.

Finally, the gauge field interpolation as we have defined it is a rather cumbersome procedure. A more economical one was given in ref. 20.]. It satisfies all the criteria we give in section 3 , with the possible exception of lattice rotational invariance, which we have not been able to prove or disprove. However, this symmetry could be restored by averaging over lattice rotations,

$$
\Gamma[U] \rightarrow \frac{\sum_{R} \Gamma\left[U^{R}\right]}{\sum_{R}},
$$

where $U^{R}$ is a lattice rotation of $U$. It is important to stress that cubic invariance is an essential ingredient to recovering a Lorentz invariant continuum limit.

\section{Conclusion.}

In this paper, we have presented a non-perturbative lattice formulation of chiral gauge theories. It provides a finite algorithm for computer simulations. The gauge fields live on a lattice with spacing $b$, taken as the defining 'cutoff' of the theory. For each gauge field configuration the chiral fermion determinant is computed by first interpolating the gauge field to a finer lattice with spacing $f \ll b$; the determinant is then constructed in the background of this interpolated field. Though it is difficult to maintain gauge invariance and eliminate the doubler modes that plague lattice fermion constructions, the loss of gauge invariance can be minimized using a separate 'hybrid' regulator for the magnitude and phase of the fermion determinant. 
The gauge non-invariance of the determinant is then of order $f^{2}$ so long as gauge anomalies cancel. The gauge field loops do not upset this suppression since their highest momenta are of order $1 / b$. There is one subtlety, that the gauge field interpolation is smooth enough to not reintroduce powers of $1 / f$ that can destroy the approximate gauge invariance. We checked that this did not happen with the choice of 'transversely continuous' interpolation made here. In the continuum limit $f \rightarrow 0$, followed by $b \rightarrow 0$, exact gauge invariance is recovered.

The price of maintaining gauge invariance is the computational expense of gauge field interpolation and calculation of large fermion determinants. We argued that in order to be insensitive to the breakage of gauge invariance in a simulation in a physical volume $L^{4}$, one would have to take

$$
f / b \sim b / L
$$

Strategies for improved efficiency were discussed including matching the twocutoff theory to a one-cutoff theory. The main computational effort would then be in doing the matching. If this is possible, the one-cutoff theory could be used for all amplitudes of interest, with the same cost as (unquenched) lattice QCD simulations!

We also explored anomalies in global currents in our lattice construction of chiral gauge theory, demonstrating the validity of the Adler-Bardeen theorem to all orders. We briefly discussed the related physics of fermion number violation, using $B+L$-violation in a simplified 'standard model' as an example.

At present, the task of using the algorithm presented here to simulate four-dimensional, non-abelian chiral gauge theories seems computationally formidable. Of course, we hope that there can be improvements made in the efficiency of the algorithm, and in the ability of computers to carry them out. In the meantime, simulation of two-dimensional chiral gauge theories seems quite feasible and provides a useful arena for testing the method.

\section{Acknowledgments}

We wish to thank S. Coleman, M. Golden, S. Hsu, S. Kachru, J.P. Leroy, O. Péne, C. Pittori and K. Rajagopal for discussions, and to Ph. Boucaud and J.L. Alonso for a careful reading of our manuscript. We are especially grateful to C. Rebbi for discussions and encouragement. Finally, we want 
to thank O. Narayan for repeatedly helping us in times of mathematical confusion.

\section{Appendix A}

In this appendix we will prove in more technical detail the general bound on one-fermion-loop lattice integrals (38), and the stricter bounds (44) for the gauge invariant parity-even contributions in $n=2,3$ and for the noninvariant parity-odd $n=3$ amplitudes.

These integrals have the form,

$$
\Gamma^{(n)}(q)=\int_{B Z(f)} d^{4} k I(q, k, f) \equiv \int_{B Z(f)} d^{4} k \frac{V(q, k, f)}{\prod_{i} P_{f}^{2}\left(l_{i}(k, q), f\right)}
$$

where the functions $P_{f}$ are the lattice fermion propagators, so

$$
\begin{array}{r}
P_{f}^{2}(k) \equiv \frac{1}{\sum_{\mu} \hat{k}_{\mu}^{2}+M(k)^{2}}, \\
M(k) \equiv \frac{2 r}{f} \sum_{\mu} \sin ^{2}\left(k_{\mu} f / 2\right) \quad \hat{k}_{\mu} \equiv \frac{1}{f} \sin _{\mu} f .
\end{array}
$$

The fermion momentum is $k \in B Z(f) \equiv\left(\frac{-\pi}{f}, \frac{\pi}{f}\right)$.

It has been proven in [20] for very general conditions on the functions $V$ and $P$, which our action satisfies, that the integrand can be bounded by a continuous function that does not depend on $f$, for all $f<f_{0}$ and $f_{0}$ finite and fixed. We define the integer $\omega$,

$$
V(q, k, f)=f^{-\omega} \tilde{V}(q f, k f)
$$

and the degree of divergence of $\mathrm{V}$ as,

$$
\lim _{\lambda \rightarrow \infty} V(q, k \lambda, f / \lambda)=K \lambda^{\nu}+O\left(\lambda^{\nu-1}\right)
$$

and $K \neq 0$. For the integrals we consider here, the following equality holds,

$$
2 I_{f}-\omega=n
$$

with $I_{f}$ the number of internal fermion lines and $n$ being the number of external boson lines. The last equality can be easily checked from the lattice Feynman rules for fermion loop integrals [13]. 
With these definitions, Lüscher's result is

$$
|V(q, k, f)|<C|q|^{(\omega-\nu)} Q^{\nu}(|k|,|q|),
$$

where $Q$ is a homogeneous polynomial of degree $\nu$ and $C$ is a constant independent of $f$. On the other hand, for each propagator we get,

$$
\left|P_{f}^{2}(l, f)\right|>C^{\prime} l^{2}
$$

where $C^{\prime}$ is some constant and $l^{2}$ is the square of the fermion momentum.

Then,

$$
\left|\Gamma^{(n)}(q)\right|<\int_{B Z(f)} d^{4} k \frac{C|q|^{(\omega-\nu)} Q^{\nu}(|k|,|q|)}{C^{\prime} \prod_{i} l_{i}(q, k)^{2}} .
$$

If $I_{f}$ is the number of internal fermion lines, we can distinguish two possibilities. If $d_{u v}<0$,

$$
d_{u v} \equiv 4+\nu-2 I_{f}<4-n
$$

the integral is convergent in the limit $f \rightarrow 0$ and by power counting for continuum integrals this implies,

$$
\left|\Gamma^{(n)}\right|<|q|^{4+\omega-2 I_{f}} \log (|q|)=|q|^{4-n} \log (|q|),
$$

where $n$ is the number of external boson lines.

In the case $d_{u v} \geq 0$, the integral (89) is divergent in the limit $f \rightarrow 0$, so it must depend on the cutoff $1 / f$. Note however, that we can separate the divergent parts from the finite part by an expansion at zero external momenta. From (89) we see that there are no infrared problems if

$$
d_{i r} \equiv 2 I_{f}-4-\omega<0
$$

but $d_{i r}=-d_{u v}+\nu-\omega \leq-d_{u v}$. Since the degree of infrared divergence increases with each derivative with respect to external momenta, while the ultraviolet $d_{u v}$ decreases, we see that for $u v$ divergent diagrams, we can Taylor expand up to $d_{u v}-1$ times or more. The local terms in the expansion are substracted and the remainder is only logarithmically divergent, and also possibly non-local. In our hybrid scheme and if the gauge anomalies cancel, there is no local counterterm except the one contained in this remainder, which is logarithmic and cannot be substracted at zero momentum. Only 
three cases need to be considered $n=2,3,4$. In the case of $n=4, d_{u v} \leq 0$ so the integral (89) is at most logarithmically dependent on the cutoff,

$$
\left|\Gamma_{0}^{(n)}\right|<C \log (|q| f) .
$$

We can easily renormalize it by substracting the same integral at zero external momenta, with an infrared cutoff $\sim 1 / b$,

$$
\delta \Gamma^{(4)}(0) \equiv \int_{B Z(b)->B Z(f)} d^{4} k I(0, k, f) .
$$

(This corresponds to the gauge coupling renormalization of eq. (18).) The remainder is then finite. This is easy to prove using the Lüscher bounds. The renormalized integral is then,

$$
\Gamma^{(4)}(q)=\int_{B Z(b)} d^{4} k I(q, k, f)+\int_{B Z(f)-B Z(b)} d^{4} k(I(q, k, f)-I(0, k, f))
$$

The integrand of the second term obviously vanishes for zero external momenta, so it can be written as a Taylor remainder of a first order expansion around zero external momenta. This remainder is of the form,

$$
R_{1}=\left.\sum_{\alpha} q_{\alpha} \int_{0}^{1} d t(1-t) \frac{\partial}{\partial w_{\alpha}} I(q+t w, k, f)\right|_{\omega=0},
$$

and now $R_{1}$ has $d_{u v}=-1$, so the second term is finite in the limit $f \rightarrow 0$, by (89). The first term obviously is too. From (95) we then find,

$$
\left|\Gamma^{(4)}\right|<C \log (|q| b),
$$

which is finite in the limit $f \rightarrow 0$ and has a logarithmic bound for large external momenta as expected.

The cases of $n=2$ and $n=3$, since they are only logarithmically divergent for the hybrid action, can be analyzed in the same way and the following bounds are found,

$$
\left|\Gamma^{(2)}\right|<C|q|^{2} \log (|q| b) ; \quad\left|\Gamma^{(3)}\right|<C|q| \log (|q| b) .
$$

However, as we saw these bounds are not good enough to prove $f$ convergence due to the discontinuities of the interpolation, and the stricter bounds (44) are needed. In the continuum, we know that gauge invariance implies that the two-point function is of the form,

$$
\Gamma_{\mu \nu}^{(2)}(q)=\left(q_{\mu} q_{\nu}-g_{\mu \nu} q^{2}\right) \Pi(q)
$$


where $\Pi(q)$ is a scalar function that behaves logarithmically for large external momenta. It is clear then that the component $\mu=\nu$ satisfies the bound (44). For finite $f$ however, a form like this is not easy to prove for the parity-even parts, because we do not have Lorentz invariance. Fortunately, the gauge invariant relations of eqs. (43) are enough. Let us start with the two-point function. Using (43) repeatedly we find,

$$
\Gamma_{\mu \mu}^{(2)}(q)=\hat{q}^{2} \frac{\Gamma_{\mu \mu}^{(2)}(q)}{\hat{q}^{2}}=\sum_{\beta \neq \mu} \hat{q}_{\beta}^{2} \frac{\Gamma_{\mu \mu}^{(2)}}{\hat{q}^{2}}-\sum_{\alpha, \beta \neq \mu} \hat{q}_{\alpha} \hat{q}_{\beta} \frac{\Gamma_{\alpha \beta}^{(2)}}{\hat{q}^{2}}
$$

where the usual definition,

$$
\hat{q}_{\alpha} \equiv \frac{e^{i q_{\alpha} f}-1}{f},
$$

has been used. Now, the right-hand-side can easily be bounded and we find the desired result,

$$
\left|\Gamma_{\mu \mu}^{(2)}(q)\right|<\sum_{\alpha, \beta}\left|q_{\alpha}\right|\left|q_{\beta}\right|\left|\Gamma_{\alpha \beta}^{(2)}\right| \frac{1}{q^{2}}<\sum_{\alpha, \beta}\left|q_{\alpha}\right|\left|q_{\beta}\right| \log (|q|), \quad \alpha, \beta \neq \mu .
$$

The last inequality has made use of eq. (98). The P-even three-point function can be analysed in the same way.

Finally, the parity-odd three point function has to be considered. In this case we do not have the help of the gauge invariance constraints (43), but on the other hand, the diagram contains an epsilon tensor. A direct inspection of the three-point function reveals the following structure,

$$
\begin{array}{r}
\Gamma_{\mu \mu \mu}^{(3)}\left(q, q^{\prime}\right) \sim f^{a b c} \epsilon_{\mu \alpha \beta \gamma}\left\{4 \bar{s}\left(q_{\beta}^{\prime}\right) \bar{s}\left(q_{\gamma}\right) F_{1}\left(q, q^{\prime}\right)-2 \bar{s}\left(q_{\gamma}\right) s\left(q_{\beta}^{\prime}\right) F_{2}\left(q, q^{\prime}\right)\right. \\
\left.+2 s\left(q_{\gamma}\right) \bar{s}\left(q_{\beta}^{\prime}\right) F_{3}\left(q, q^{\prime}\right)-s\left(q_{\gamma}\right) s\left(q_{\beta}^{\prime}\right) F_{4}\left(q, q^{\prime}\right)\right\},
\end{array}
$$

where

$$
\bar{s}(q) \equiv \frac{\sin ^{2}(q f / 2)}{f^{2}} s(q) \equiv \frac{\sin (q f)}{f},
$$

and the functions $F$ are lattice integrals with smaller degree of divergence than the original integral, for which the following bounds hold,

$$
\begin{aligned}
\left|F_{1}\left(q, q^{\prime}\right)\right|<\frac{1}{|q|^{3}} \log (|q|),\left|F_{2,3}\left(q, q^{\prime}\right)\right| & <\frac{1}{|q|^{2}} \log (|q|) \\
\left|F_{4}\left(q, q^{\prime}\right)\right| & <\frac{1}{|q|} \log (|q|) .
\end{aligned}
$$


Using $|\bar{s}(q)|<C|q|^{2},|s(q)|<C|q|$, it is then straightforward to check that

$$
\left|\Gamma_{\mu \mu \mu}^{(3)}\left(q, q^{\prime}\right)\right|<\left|q_{\alpha}\right| \log (|q|), \quad \alpha \neq \mu,
$$

This concludes the proof of the bound (43) for the P-odd three-point function.

\section{References}

[1] For examples of other recent activity see the articles appearing in Nucl.Phys. B(Proc.Suppl) 34(1994).

[2] R. Flume and D. Wyler, Phys. Lett.108B(1982)317.

[3] G. 't Hooft, Phys.Lett.B349(1995)491.

[4] M. Gockeler, G. Schierholz, Nucl.Phys. B(Proc.Suppl) 29B,C(1992) 114; Nucl.Phys. B(Proc.Suppl) 30(1993) 609.

[5] S. Hsu, hep-th/9503058.

[6] A. Kronfeld, FERMILAB-PUB-95/073-T or hep-lat/9504007.

[7] H.B. Nielsen and H. Ninomiya, Nucl. Phys. B185(1981)20,(E) B195(1982) 541 and Nucl. Phys. B193(1981)173.

[8] L.H. Karsten and J. Smit. Nucl. Phys. B183(1981) 103-140.

[9] A. Borrelli, L. Maiani, G.C. Rossi, R. Sisto and M. Testa, Nucl. Phys. B333(1990) 335-356.

[10] L. Maiani, G.C. Rossi and M. Testa, Phys. Lett. B292(1992) 397.

[11] J.L. Alonso, Ph. Boucaud, J.L. Cortes and E. Rivas, Mod. Phys. Lett. A5(1990) 275; Nucl.Phys. B(Proc.Suppl) 17(1990) 461.

[12] J. Wess and B. Zumino, Phys. Lett. 37B(1971) 95; W.A. Bardeen and B. Zumino, Nucl. Phys. B244(1984) 421-453.

[13] See for instance Lattice Gauge Theories by H.J. Rothe, World Scientific Lecture Notes in Physics-Vol 431992.

[14] M. Golterman and D. Petcher, Phys. Lett. 225(1989) 159. 
[15] L. Alvarez-Gaumé and S. Della Pietra, in Recent Developments in Quantum Field Theory, eds. J. Ambjorn, B.J. Durhuus and J.L. Petersen, North-Holland (1985).

[16] G.T. Bodwin and E.V. Kovacs, Nucl.Phys. B(Proc.Suppl) 30(1993) 617.

[17] E. Witten, Phys. Lett. 117B(1982) 324.

[18] M. Lüscher, Comm. Math. 85, 39-48 (1982).

[19] T. Reisz, Comm. Math. 117, (1988a) 79; 117(1988b) 639; 116 (1988c) 81.

[20] M. Lüscher, Lectures at Summer School on Fields, Strings and Critical Phenomena, Les Houches (1988).

[21] J. Smit, Nucl.Phys. B(Proc.Suppl) 29B,C(1992) 83.

[22] S. Adler and W.A. Bardeen, Phys. Rev. 182 (1969) 1517.

[23] H. Kawai, R. Nakayama and K. Seo, Nucl. Phys. B189, 40 (1981).

[24] R. Narayanan and H. Neuberger, Nucl. Phys. B443(1995) 305.

[25] G. 't Hooft, Phys. Rev. Lett. 37 (1976) 8.

[26] V. Zakharov, Nucl. Phys. B371 (1992) 637-658. M. Porrati, Nucl. Phys. B347(1990) 371-393. V.V. Khoze and A. Ringwald, Nucl. Phys. B355(1991) 351.

[27] K. Fujikawa, Phys. Rev D21 (1980) 2848 and 29 (1984) 285.

[28] M.F. Atiyah and I.M. Singer, Ann. Math. 87, 484 (1968).

[29] M. Göckeler, A. Kronfeld, G. Schierholz and U.J.Wiese, Nucl. Phys. B404(1993) 839. 